\title{
Convergence of an ADI splitting for Maxwell's equations
}

\author{
Marlis Hochbruck • Tobias Jahnke • \\ Roland Schnaubelt
}

April 2014

\begin{abstract}
The convergence of an alternating direction implicit method for Maxwell's equations on product domains is investigated. Unlike the classical Yee scheme and most other integrators proposed in the literature, this method is both unconditionally stable and computationally cheap. We prove second-order convergence of the time-discretization in the framework of operator semigroup theory. In contrast to formal considerations based on Taylor expansions, our convergence analysis respects the unboundedness of the involved differential operators. The proofs are based on results concerning the regularity of the Cauchy problems, which then allow to apply an abstract convergence proof by Hansen and Ostermann [13].
\end{abstract}

Keywords Maxwell's equations, alternating direction implicit method, Peaceman-Rachford splitting, well-posedness, regularity, error analysis, time integration, semigroups

Mathematics Subject Classification (2000) Primary: 65M12. Secondary: 35Q61, 47D03, 65J10.

\section{Introduction}

Maxwell's equations provide the foundation for the modern theory of electromagnetism, and solving these equations numerically is a crucial task in the analysis and design of antennas, photonic cristals, waveguides, and mobile communication devices. In the majority of simulations, the solution of Maxwell's equations is approximated with finite-difference time-domain methods (cf. [22]). Within this class, the Yee scheme [27] is particularly popular,

Department of Mathematics, Karlsruhe Institute of Technology, 76128 Karlsruhe, Germany. E-mail: marlis.hochbruck@kit.edu, tobias.jahnke@kit.edu, schnaubelt@kit.edu. Corresponding author: R. Schnaubelt, Phone: +49-721-60848955, Fax: +49-721-60847650. 
but since this method is explicit, instability can only be avoided if a sufficiently small step size is chosen. This can seriously affect the efficiency of the method; cf. [22]. On the other hand, using an implicit and unconditionally stable Runge-Kutta method for the time integration may decrease the necessary number of time-steps, but the price to pay is a large linear system which has to be solved in each step. Thus, the total numerical costs of such an implicit method is often not significantly smaller than the computational work of the Yee scheme.

A major breakthrough for the simulation of Maxwell equations posed on a cuboid or on the whole of $\mathbb{R}^{3}$ was achieved around 2000 in $[17 ; 18 ; 28]$, where an unconditionally stable and computationally efficient alternating direction implicit (ADI) method was proposed. The main idea is, roughly speaking, to decompose the Maxwell operator into two parts and to propagate the associated sub-flows in such a way that the implicitness is reduced to one-dimensional problems. Hence, instead of the large linear systems with large bandwidth arising in the discretization of the full $3 \mathrm{~d}$ problem, only small linear systems with tridiagonal matrices have to be solved in each time-step. This invention raised a lot of interest, and a large number of follow-up papers can be found in the literature, see, e.g., $[7 ; 10 ; 9 ; 11]$ and references therein. Moreover, the operator splitting approach was modified and extended for the construction of new methods by composition; cf. $[2 ; 8 ; 14 ; 15 ; 24 ; 25 ; 26]$ and Chapter 18 in [22]. Splitting and composition methods for ordinary differential equations are discussed in Section II.4-II.5 in [12].

It is well known that the method proposed in $[17 ; 28]$ is formally of second order in time and space. Most of the convergence results found in the literature use Taylor expansion of the exact solution to prove second order error bounds. Ultimately, this leads to bounds where the leading error term depends on the norm of the finite difference matrices used in the spatial discretization. However, since these matrices approximate unbounded differential operators, their norm tends to infinity when the spatial mesh width tends to zero. Hence, such an analysis only proves that the method converges with order two in time if a fixed spatial mesh is considered. The argument does not reveal whether or not the accuracy of the time integration is reduced when the spatial approximation is refined.

The main goal of this paper is to prove that under suitable regularity conditions the second-order convergence in time is indeed not affected by the spatial discretization. To this end, we prove an error bound for the semidiscretization in a framework of operator semigroup theory which takes into account that all operators involving spatial derivatives are unbounded. Our error analysis is based on an explicit formula for the global error, which was already used in [13] in a different setting. To estimate the terms in our error formula, we need the skew-adjointness both of the operator governing the Maxwell equation and of the operators arising in the splitting. Here, it is crucial to choose the correct boundary conditions for the splitted problems. It remains one core term, which has to be treated by means of a detailed regularity analysis given in Lemmas 3.6 and 3.7. 
In Section 2 we introduce Maxwell's equations on $\mathbb{R}^{3}$ and on a cuboid, and we formulate the method from [28] as a Peaceman-Rachford splitting method. The computational advantage of this approach is explained in Section 2.3. Section 3 is devoted to the analysis of Maxwell's equations. We describe and investigate in detail the analytical setting and establish the necessary regularity results. Based on this analytical background, we present our error analysis of the semi-discretization on $\mathbb{R}^{3}$ and on a cuboid in Section 4 (cf. Theorems 4.2 and 4.5). The convergence results are confirmed by numerical examples which illustrate how the accuracy is affected if the regularity of the initial data or of the coefficients is low. Finally, we present the proofs of two technical lemmas in the appendix.

Notation. Throughout the article, the Euclidean scalar product on $\mathbb{R}^{3}$ is denoted by $x \cdot y$. We write $Y \hookrightarrow Z$ if a Banach space $Y$ is continuously embedded into a Banach space $Z$. The domain $D(A)$ of a linear operator $A$ is endowed with the graph norm $\|x\|+\|A x\|$. The domain of a product of linear operators is defined by

$$
D(A B)=\{x \in D(B) \mid B x \in D(A)\}
$$

and recursively for more factors such as $A^{n}$. We use real valued function spaces. All constants that only depend on the coefficients $\varepsilon$ and $\mu$ are denoted by $c$.

Acknowledgement. We thank the referees for useful comments which in particular led to a simplification of the proof of Theorem 4.2.

\section{The ADI splitting for Maxwell's equations}

\subsection{Maxwell's equations}

We consider linear Maxwell's equations without sources on $\mathbb{R}^{3}$

$$
\begin{aligned}
\partial_{t} \mathbf{E}(t) & =\frac{1}{\varepsilon} \operatorname{rot} \mathbf{H}(t), & & t \in \mathbb{R}, x \in \mathbb{R}^{3}, \\
\partial_{t} \mathbf{H}(t) & =-\frac{1}{\mu} \operatorname{rot} \mathbf{E}(t), & & t \in \mathbb{R}, x \in \mathbb{R}^{3}, \\
\operatorname{div} \varepsilon \mathbf{E}(t) & =0, \quad \operatorname{div} \mu \mathbf{H}(t)=0, & & t \in \mathbb{R}, x \in \mathbb{R}^{3}, \\
\mathbf{E}(0) & =\mathbf{E}^{0}, \quad \mathbf{H}(0)=\mathbf{H}^{0}, & & x \in \mathbb{R}^{3},
\end{aligned}
$$

and on the cuboid $Q=\left(a_{1}^{-}, a_{1}^{+}\right) \times\left(a_{2}^{-}, a_{2}^{+}\right) \times\left(a_{3}^{-}, a_{3}^{+}\right) \subseteq \mathbb{R}^{3}$

$$
\begin{aligned}
\partial_{t} \mathbf{E}(t) & =\frac{1}{\varepsilon} \operatorname{rot} \mathbf{H}(t), & & t \in \mathbb{R}, x \in Q, \\
\partial_{t} \mathbf{H}(t) & =-\frac{1}{\mu} \operatorname{rot} \mathbf{E}(t), & & t \in \mathbb{R}, x \in Q, \\
\operatorname{div} \varepsilon \mathbf{E}(t) & =0, \quad \operatorname{div} \mu \mathbf{H}(t)=0, & & t \in \mathbb{R}, x \in Q, \\
\mathbf{E}(t) \times \nu & =0, \quad \mu \mathbf{H}(t) \cdot \nu=0, & & t \in \mathbb{R}, x \in \partial Q, \\
\mathbf{E}(0) & =\mathbf{E}^{0}, \quad \mathbf{H}(0)=\mathbf{H}^{0}, & & x \in Q,
\end{aligned}
$$

with a perfectly conducting boundary. The electric field $\mathbf{E}=\mathbf{E}(t, x)$ and the magnetic field $\mathbf{H}=\mathbf{H}(t, x)$ vary in time and space, but the spatial variable 
$x$ will usually be omitted. The corresponding initial fields are $\mathbf{E}^{0} \in L^{2}(\Omega)^{3}$ and $\mathbf{H}^{0} \in L^{2}(\Omega)^{3}$, where $\Omega \in\left\{\mathbb{R}^{3}, Q\right\}$. We assume that the permittivity $\varepsilon \in L^{\infty}(\Omega)$ and the permeability $\mu \in L^{\infty}(\Omega)$ are given functions which satisfy $\varepsilon(x), \mu(x) \geq \delta>0$ for a constant $\delta>0$. In the boundary conditions of $(2), \nu$ is the outer unit normal on the boundary $\partial Q$ (defined outside the edges). The differential operators and boundary conditions are understood in the sense of distributions and traces, respectively. It is known that these equations are well-posed in $L^{2}(\Omega)^{6}$, see, e.g., Theorem 8.5 in [16] or Section XVII.B.4.4 in [5]. More precisely, the Maxwell operator

$$
M=\left(\begin{array}{cc}
0 & \frac{1}{\varepsilon} \operatorname{rot} \\
-\frac{1}{\mu} \operatorname{rot} & 0
\end{array}\right)
$$

is skew-adjoint on a certain subspace of $L^{2}(\Omega)^{6}$ if we include the divergence and boundary conditions in a suitable way in this subspace and in the domain of $M$, and if we equip $L^{2}(\Omega)^{6}$ with the scalar product corresponding to the energy of the fields. Moreover, the divergence conditions for $\varepsilon \mathbf{E}$ and for $\mu \mathbf{H}$ and the boundary condition for $\mu \mathbf{H}$ follow from the other equations in (1) or (2) if the initial fields satisfy these conditions, see Propositions 3.1 and 3.5.

However, it is hard to find a detailed proof for these results in the present generality. Moreover, the framework of the well-posedness results is needed for our error analysis. We have thus included the arguments in Section 3, which focuses on additional regularity properties of $M$. The proofs are postponed to the appendix.

\subsection{ADI splitting scheme}

The time discretization proposed in [28] is based on the idea to split the differential operator rot into

$$
\operatorname{rot}=C_{1}-C_{2} \quad \text { with } \quad C_{1}=\left(\begin{array}{ccc}
0 & 0 & \partial_{2} \\
\partial_{3} & 0 & 0 \\
0 & \partial_{1} & 0
\end{array}\right), \quad C_{2}=\left(\begin{array}{ccc}
0 & \partial_{3} & 0 \\
0 & 0 & \partial_{1} \\
\partial_{2} & 0 & 0
\end{array}\right)
$$

and to define

$$
A=\left(\begin{array}{cc}
0 & \frac{1}{\varepsilon} C_{1} \\
\frac{1}{\mu} C_{2} & 0
\end{array}\right) \quad \text { and } \quad B=\left(\begin{array}{cc}
0 & -\frac{1}{\varepsilon} C_{2} \\
-\frac{1}{\mu} C_{1} & 0
\end{array}\right)
$$

The operators $A$ and $B$ act on $L^{2}(\Omega)^{6}$. They are endowed with the "maximal" domains

$$
\begin{aligned}
& D_{\mathbb{R}^{3}}(A)=\left\{(u, v) \in L^{2}\left(\mathbb{R}^{3}\right)^{6} \mid\left(C_{1} v, C_{2} u\right) \in L^{2}\left(\mathbb{R}^{3}\right)^{6}\right\}, \\
& D_{\mathbb{R}^{3}}(B)=\left\{(u, v) \in L^{2}\left(\mathbb{R}^{3}\right)^{6} \mid\left(C_{2} v, C_{1} u\right) \in L^{2}\left(\mathbb{R}^{3}\right)^{6}\right\}
\end{aligned}
$$

on the full space $\mathbb{R}^{3}$ and with "partial" Dirichlet boundary conditions 


$$
\begin{aligned}
& D_{Q}(A)=\left\{(u, v) \in L^{2}(Q)^{6} \mid\left(C_{1} v, C_{2} u\right) \in L^{2}(Q)^{6},\right. \\
& \left.u_{1}=0 \text { on } \Gamma_{2}^{ \pm}, u_{2}=0 \text { on } \Gamma_{3}^{ \pm}, u_{3}=0 \text { on } \Gamma_{1}^{ \pm}\right\}, \\
& D_{Q}(B)=\left\{(u, v) \in L^{2}(Q)^{6} \mid\left(C_{2} v, C_{1} u\right) \in L^{2}(Q)^{6},\right. \\
& \left.u_{1}=0 \text { on } \Gamma_{3}^{ \pm}, u_{2}=0 \text { on } \Gamma_{1}^{ \pm}, u_{3}=0 \text { on } \Gamma_{2}^{ \pm}\right\}
\end{aligned}
$$

on $Q$. Often we will omit the subscript indicating the spatial domain. Here and below $\Gamma_{j}^{-}$and $\Gamma_{j}^{+}$are the open faces of $Q$ given by $x_{j}=a_{j}^{-}$and $x_{j}=a_{j}^{+}$, respectively, for $j=1,2,3$. Note that the boundary conditions in $D_{Q}(A)$ and $D_{Q}(B)$ are well defined since the corresponding partial derivatives are square integrable. The domains of $A$ and $B$ are chosen such that $D(A) \cap D(B) \subseteq$ $D(M)$ and $A w+B w=M w$ for $w \in D(A) \cap D(B)$ and for both $\Omega=\mathbb{R}^{3}$ and $\Omega=Q$. For each of the two cases, the domain of $M$ will be defined in the next section. We remark that $A$ and $B$ do neither respect the divergence condition nor the magnetic boundary condition of Maxwell's equations.

For a step size $\tau>0$ and $w \in D(B)$, the ADI splitting method proposed in [28] can now be formulated as

$$
S_{\tau} w=\left(I-\frac{\tau}{2} B\right)^{-1}\left(I+\frac{\tau}{2} A\right)\left(I-\frac{\tau}{2} A\right)^{-1}\left(I+\frac{\tau}{2} B\right) w .
$$

Hence, this scheme is a special case of the Peaceman-Rachford method, cf. [13]. We will show in Section 4 that $A$ and $B$ are skew-adjoint (cf. Lemmas 4.1 and $4.3)$ and thus the above inverses exist. Moreover, this implies $\|\left(I+\frac{\tau}{2} A\right)(I-$ $\left.\frac{\tau}{2} A\right)^{-1}\|=\|\left(I+\frac{\tau}{2} B\right)\left(I-\frac{\tau}{2} B\right)^{-1} \|=1$ in a suitable norm, and since the approximation $w_{n}$ obtained after $n$ steps is given by

$$
\begin{aligned}
S_{\tau}^{n} w & =\left(I-\frac{\tau}{2} B\right)^{-1} P_{\tau}^{n-1}\left(I+\frac{\tau}{2} A\right)\left(I-\frac{\tau}{2} A\right)^{-1}\left(I+\frac{\tau}{2} B\right) w \\
\text { with } P_{\tau} & =\left(I+\frac{\tau}{2} A\right)\left(I-\frac{\tau}{2} A\right)^{-1}\left(I+\frac{\tau}{2} B\right)\left(I-\frac{\tau}{2} B\right)^{-1},
\end{aligned}
$$

it follows that

$$
\left\|S_{\tau}^{n} w\right\| \leq\left\|\left(I-\frac{\tau}{2} B\right)^{-1}\right\| \cdot\left\|\left(I+\frac{\tau}{2} B\right) w\right\| .
$$

Since the right-hand side is independent of $n$, the method is unconditionally stable. This was proved in a similar way in [7] for matrices instead of operators. Alternative proofs of the unconditional stability can be found, e.g., in [15].

Our main Theorems 4.2 and 4.5 say that

the ADI splitting scheme $S_{\tau}^{n}\left(\mathbf{E}^{0}, \mathbf{H}^{0}\right)$ converges quadratically in $L^{2}(\Omega)^{6}$ to the solutions of (1), resp. (2), if $\mathbf{E}^{0}, \mathbf{H}^{0}, \varepsilon$ and $\mu$ are sufficiently regular.

2.3 Efficient formulation of the ADI splitting scheme on $\mathbb{R}^{3}$

As the definition (4) indicates, each time step of the ADI splitting method involves two implicit substeps corresponding to the two inverses. In [28], the approximations

$$
\begin{aligned}
\left(\mathbf{E}^{n}, \mathbf{H}^{n}\right) & =S_{\tau}^{n}\left(\mathbf{E}^{0}, \mathbf{H}^{0}\right) \in D(B) \quad \text { and } \\
\left(\mathbf{E}^{n+\frac{1}{2}}, \mathbf{H}^{n+\frac{1}{2}}\right) & =\left(I-\frac{\tau}{2} A\right)^{-1}\left(I+\frac{\tau}{2} B\right)\left(\mathbf{E}^{n}, \mathbf{H}^{n}\right) \in D(A), \quad n \in \mathbb{N},
\end{aligned}
$$


were replaced by equivalent ones in such a way that the linear systems arising from the implicit parts can be solved in a very efficient way. This idea is the main advantage of the method over most other implicit methods.

We first derive the equivalent scheme in $\mathbb{R}^{3}$. The first half step given by (5) can be written as

$$
\begin{aligned}
\mathbf{E}^{n+\frac{1}{2}} & =\mathbf{E}^{n}-\frac{\tau}{2 \varepsilon} C_{2} \mathbf{H}^{n}+\frac{\tau}{2 \varepsilon} C_{1} \mathbf{H}^{n+\frac{1}{2}}, \\
\mathbf{H}^{n+\frac{1}{2}} & =\mathbf{H}^{n}-\frac{\tau}{2 \mu} C_{1} \mathbf{E}^{n}+\frac{\tau}{2 \mu} C_{2} \mathbf{E}^{n+\frac{1}{2}} .
\end{aligned}
$$

We eliminate $\mathbf{H}^{n+\frac{1}{2}}$ by inserting the second equality into the first to deduce

$$
\begin{aligned}
\mathbf{E}^{n+\frac{1}{2}} & =\mathbf{E}^{n}-\frac{\tau}{2 \varepsilon} C_{2} \mathbf{H}^{n}+\frac{\tau}{2 \varepsilon} C_{1}\left(\mathbf{H}^{n}-\frac{\tau}{2 \mu} C_{1} \mathbf{E}^{n}+\frac{\tau}{2 \mu} C_{2} \mathbf{E}^{n+\frac{1}{2}}\right) \\
& =\mathbf{E}^{n}+\frac{\tau}{2 \varepsilon}\left(C_{1}-C_{2}\right) \mathbf{H}^{n}-\frac{\tau^{2}}{4 \varepsilon} C_{1} \mu^{-1} C_{1} \mathbf{E}^{n}+\frac{\tau^{2}}{4 \varepsilon} C_{1} \mu^{-1} C_{2} \mathbf{E}^{n+\frac{1}{2}} .
\end{aligned}
$$

Here one applies partial derivatives to functions in $L^{2}\left(\mathbb{R}^{3}\right)$ so that from now on the equations for $\mathbf{E}^{n+\frac{1}{2}}$ and $\mathbf{E}^{n+1}$ hold in $H^{-1}\left(\mathbb{R}^{3}\right)^{3}$. This leads to the equivalent scheme

$$
\begin{aligned}
\left(I-\frac{\tau^{2}}{4 \varepsilon} C_{1} \mu^{-1} C_{2}\right) \mathbf{E}^{n+\frac{1}{2}} & =\mathbf{E}^{n}+\frac{\tau}{2 \varepsilon}\left(C_{1}-C_{2}\right) \mathbf{H}^{n}-\frac{\tau^{2}}{4 \varepsilon} C_{1} \mu^{-1} C_{1} \mathbf{E}^{n}, \\
\mathbf{H}^{n+\frac{1}{2}} & =\mathbf{H}^{n}-\frac{\tau}{2 \mu} C_{1} \mathbf{E}^{n}+\frac{\tau}{2 \mu} C_{2} \mathbf{E}^{n+\frac{1}{2}} .
\end{aligned}
$$

Similarly, the second half step can be transformed into

$$
\begin{aligned}
\left(I-\frac{\tau^{2}}{4 \varepsilon} C_{2} \mu^{-1} C_{1}\right) \mathbf{E}^{n+1} & =\mathbf{E}^{n+\frac{1}{2}}+\frac{\tau}{2 \varepsilon}\left(C_{1}-C_{2}\right) \mathbf{H}^{n+\frac{1}{2}}-\frac{\tau^{2}}{4 \varepsilon} C_{2} \mu^{-1} C_{2} \mathbf{E}^{n+\frac{1}{2}}, \\
\mathbf{H}^{n+1} & =\mathbf{H}^{n+\frac{1}{2}}+\frac{\tau}{2 \mu} C_{2} \mathbf{E}^{n+\frac{1}{2}}-\frac{\tau}{2 \mu} C_{1} \mathbf{E}^{n+1} .
\end{aligned}
$$

The implicit parts are thus reduced to the products

$$
\begin{aligned}
C_{1} \mu^{-1} C_{2} & =\left(\begin{array}{ccc}
\partial_{2} \mu^{-1} \partial_{2} & 0 & 0 \\
0 & \partial_{3} \mu^{-1} \partial_{3} & 0 \\
0 & 0 & \partial_{1} \mu^{-1} \partial_{1}
\end{array}\right), \\
C_{2} \mu^{-1} C_{1} & =\left(\begin{array}{ccc}
\partial_{3} \mu^{-1} \partial_{3} & 0 & 0 \\
0 & \partial_{1} \mu^{-1} \partial_{1} & 0 \\
0 & 0 & \partial_{2} \mu^{-1} \partial_{2}
\end{array}\right),
\end{aligned}
$$

which are diagonal, such that the implicit steps are fully decoupled. Since each of the differential operators on the diagonal acts only on one of the spatial directions, the spatial discretization of (6) and (7) involves linear systems which are considerably smaller than the corresponding systems in the direct formulation (5). In Section 4.3 we extend this derivation to the case of the cuboid $Q$ which is more involved due to the boundary conditions. We will see that the approximations given by (5) satisfy (6) and (7) in a weak sense. 


\section{Analysis of Maxwell's equations}

In this section we show the well-posedness of the Maxwell systems (1) and (2) and establish certain additional regularity properties. Throughout, $\Omega$ denotes an open set in $\mathbb{R}^{3}$. We are given $\varepsilon, \mu \in L^{\infty}(\Omega)$ with $\varepsilon, \mu \geq \delta>0$ for a constant $\delta>0$. The state space $X=L^{2}(\Omega)^{6}$ is endowed with the weighted scalar product given by

$$
((\mathbf{E}, \mathbf{H}) \mid(u, v))_{X}=(\mathbf{E} \mid u)_{\varepsilon}+(\mathbf{H} \mid v)_{\mu}=\int_{\Omega} \varepsilon \mathbf{E} \cdot u \mathrm{~d} x+\int_{\Omega} \mu \mathbf{H} \cdot v \mathrm{~d} x
$$

which is equivalent to the standard scalar product in $L^{2}(\Omega)^{6}$ by our assumptions on $\varepsilon$ and $\mu$. We will further need the spaces

$$
\begin{aligned}
& H(\operatorname{rot})=H(\operatorname{rot}, \Omega)=\left\{u \in L^{2}(\Omega)^{3} \mid \operatorname{rot} u \in L^{2}(\Omega)^{3}\right\}, \\
& H(\operatorname{div})=H(\operatorname{div}, \Omega)=\left\{u \in L^{2}(\Omega)^{3} \mid \operatorname{div} u \in L^{2}(\Omega)\right\} .
\end{aligned}
$$

Since the differential operators are defined in distributional sense, it is straightforward to verify that rot and div are closed in $L^{2}(\Omega)^{3}$ if endowed with their "maximal" domains $H(\operatorname{rot}, \Omega)$ and $H(\operatorname{div}, \Omega)$, respectively. These spaces are thus complete if equipped with the graph norm of the respective operators. Often we will omit the spatial domain in the notation. We point out that $u \in H$ (rot) means that, e.g., $\partial_{2} u_{3}-\partial_{3} u_{2}$ belongs to $L^{2}(\Omega)$ though the partial derivatives $\partial_{2} u_{3}$ and $\partial_{3} u_{2}$ do not need to be functions.

3.1 Well-posedness and regularity on the full space $\mathbb{R}^{3}$

We will first treat the full space setting $\left(\Omega=\mathbb{R}^{3}\right)$ separately since this case is less technical and here the line of arguments is quite transparent. We first note that the space of test functions $C_{c}^{\infty}\left(\mathbb{R}^{3}\right)^{3}$ is dense in $H\left(\operatorname{rot}, \mathbb{R}^{3}\right)$ and $H\left(\operatorname{div}, \mathbb{R}^{3}\right)$, which can be seen by standard (scalar) cutoff functions and mollifiers. The equations

$$
\begin{aligned}
\int_{\mathbb{R}^{3}} \operatorname{rot} u \cdot \varphi \mathrm{d} x & =\int_{\mathbb{R}^{3}} u \cdot \operatorname{rot} \varphi \mathrm{d} x \\
\int_{\mathbb{R}^{3}} \psi \operatorname{div} v \mathrm{~d} x & =-\int_{\mathbb{R}^{3}} v \cdot \nabla \psi \mathrm{d} x
\end{aligned}
$$

hold for test functions and hence for all $u, \varphi \in H\left(\operatorname{rot}, \mathbb{R}^{3}\right), v \in H\left(\operatorname{div}, \mathbb{R}^{3}\right)$, and $\psi \in H^{1}\left(\mathbb{R}^{3}\right)$. To treat the Maxwell system, we further need the closed subspace

$$
X_{0}=\left\{(\mathbf{E}, \mathbf{H}) \in L^{2}\left(\mathbb{R}^{3}\right)^{6} \mid \operatorname{div}(\varepsilon \mathbf{E})=\operatorname{div}(\mu \mathbf{H})=0\right\}
$$

of $X$. Recall the expression of the Maxwell operator $M$ from (3). We endow this operator on $X$ and its restriction $M_{0}$ to $X_{0}$ with the domains

$$
D(M)=D_{\mathbb{R}^{3}}(M)=H\left(\operatorname{rot}, \mathbb{R}^{3}\right)^{2}, \quad D\left(M_{0}\right)=D_{\mathbb{R}^{3}}\left(M_{0}\right)=D_{\mathbb{R}^{3}}(M) \cap X_{0} .
$$


Here and below we usually omit the subscript indicating the spatial domain. Actually, only the operator $M_{0}$ is physically relevant, but sometimes also $M$ is useful in the analysis. We next show the well-posedness of (1).

Proposition 3.1. Let $\Omega=\mathbb{R}^{3}$ and $\varepsilon, \mu \in L^{\infty}\left(\mathbb{R}^{3}\right)$ satisfy $\varepsilon, \mu \geq \delta>0$ for a constant $\delta>0$. Then the Maxwell operators $M$ and $M_{0}$ are skew-adjoint on $X$ and $X_{0}$, and thus generate unitary $C_{0}$-groups $T(t)=e^{t M}$ on $X$ and $T_{0}(t)=$ $e^{t M_{0}}$ on $X_{0}$ for $t \in \mathbb{R}$, respectively. Therefore, for each $\left(\mathbf{E}^{0}, \mathbf{H}^{0}\right) \in D\left(M_{0}\right)$ we have a unique solution $(\mathbf{E}, \mathbf{H}) \in C^{1}\left(\mathbb{R} ; L^{2}\left(\mathbb{R}^{3}\right)^{6}\right) \cap C\left(\mathbb{R} ; D\left(M_{0}\right)\right)$ of $(1)$.

Moreover, $M$ maps $D(M)$ into $X_{0}$. Hence, $D\left(M_{0}^{j}\right)=D\left(M^{j}\right) \cap X_{0}$ and the operators $T_{0}(t)$ and $\left(\lambda I-M_{0}\right)^{-1}$ are the restrictions of $T(t)$ and $(\lambda I-M)^{-1}$ to $X_{0}$, for all $j \in \mathbb{N}, t \in \mathbb{R}$, and $\lambda \in \mathbb{R} \backslash\{0\}$.

Proof. We first note that $M$ and $M_{0}$ are closed because of the closedness of rot and div. To show the skew-symmetry of $M$, we take $w=(\mathbf{E}, \mathbf{H})$ and $w^{\prime}=\left(\mathbf{E}^{\prime}, \mathbf{H}^{\prime}\right)$ in $D(M)$. The integration by parts formula (10) then implies

$$
\begin{aligned}
\left(M w \mid w^{\prime}\right)_{X} & =\left(\frac{1}{\varepsilon} \operatorname{rot} \mathbf{H} \mid \mathbf{E}^{\prime}\right)_{\varepsilon}-\left(\frac{1}{\mu} \operatorname{rot} \mathbf{E} \mid \mathbf{H}^{\prime}\right)_{\mu} \\
& =\int_{\mathbb{R}^{3}} \operatorname{rot} \mathbf{H} \cdot \mathbf{E}^{\prime} \mathrm{d} x-\int_{\mathbb{R}^{3}} \operatorname{rot} \mathbf{E} \cdot \mathbf{H}^{\prime} \mathrm{d} x \\
& =\int_{\mathbb{R}^{3}} \mathbf{H} \cdot \operatorname{rot} \mathbf{E}^{\prime} \mathrm{d} x-\int_{\mathbb{R}^{3}} \mathbf{E} \cdot \operatorname{rot} \mathbf{H}^{\prime} \mathrm{d} x \\
& =-\left(\mathbf{H} \mid-\frac{1}{\mu} \operatorname{rot} \mathbf{E}^{\prime}\right)_{\mu}-\left(\mathbf{E} \mid \frac{1}{\varepsilon} \operatorname{rot} \mathbf{H}^{\prime}\right)_{\varepsilon} \\
& =-\left(w \mid M w^{\prime}\right)_{X},
\end{aligned}
$$

and analogously for $M_{0}$.

By standard spectral theory, e.g., [20, Corollary to Theorem VIII.3], the operator $M$ is skew-adjoint if $I \pm M$ has dense range. Skew-adjointness then implies the assertions about generation and well-posedness in view of Stone's theorem [20, Theorem VIII.8]. For given $(f, g) \in X$ we have to solve the equations

$$
\mathbf{E} \pm \frac{1}{\varepsilon} \operatorname{rot} \mathbf{H}=f, \quad \mathbf{H} \mp \frac{1}{\mu} \operatorname{rot} \mathbf{E}=g
$$

with unknowns $\mathbf{E}, \mathbf{H} \in H$ (rot). It can be assumed that $g \in H$ (rot) because $H$ (rot) is dense in $X$. Formally inserting the second equation of (11) into the first one, we obtain the problem

$$
\varepsilon \mathbf{E}+\operatorname{rot}\left(\frac{1}{\mu} \operatorname{rot} \mathbf{E}\right)=\varepsilon f \mp \operatorname{rot} g=: h \in L^{2}\left(\mathbb{R}^{3}\right)^{3} .
$$

To solve this problem, we consider the symmetric bilinear form

$$
a(u, v)=\int_{\mathbb{R}^{3}}\left(\varepsilon u \cdot v+\frac{1}{\mu} \operatorname{rot} u \cdot \operatorname{rot} v\right) \mathrm{d} x
$$

on $H$ (rot). Observe that $a$ is continuous and coercive. The Lax-Milgram lemma thus yields the existence of a field $\mathbf{E} \in H$ (rot) such that

$$
\int_{\mathbb{R}^{3}}\left(\varepsilon \mathbf{E} \cdot v+\frac{1}{\mu} \operatorname{rot} \mathbf{E} \cdot \operatorname{rot} v\right) \mathrm{d} x=\int_{\mathbb{R}^{3}} h \cdot v \mathrm{~d} x
$$


holds for all $v \in H$ (rot). Since $h-\varepsilon \mathbf{E} \in L^{2}\left(\mathbb{R}^{3}\right)^{3}$, this fact implies that $\operatorname{rot}\left(\frac{1}{\mu} \operatorname{rot} \mathbf{E}\right) \in L^{2}\left(\mathbb{R}^{3}\right)^{3}$ and that $\mathbf{E}$ satisfies (12). If we now define $\mathbf{H} \in H$ (rot) by the second equation in (11), we obtain a solution $(\mathbf{E}, \mathbf{H}) \in D(M)$ of (11), as asserted.

Observe that div rot $=0$ holds also in a distributional sense. If $(f, g)$ in (11) belongs to $X_{0}$, we thus infer $(\mathbf{E}, \mathbf{H}) \in D(M) \cap X_{0}=D\left(M_{0}\right)$. Hence, $M_{0}$ is skew-adjoint in $X_{0}$. We further have $M D(M) \subseteq X_{0}$, which in turn yields the assertions about the powers and the resolvent. The identity $T_{0}(t)=$ $\left.T(t)\right|_{X_{0}}$ then follows from the resolvent approximation of the semigroups, see Corollay III.5.5 in [6].

Our approach relies on additional regularity properties of $D\left(M_{0}^{2}\right)$, proved in the following lemma. In principle this result is known, cf. Corollary IX.1.8 in [4], but we give the short and instructive proof for completeness. We write $f \in L^{p}\left(\mathbb{R}^{3}\right)+L^{q}\left(\mathbb{R}^{3}\right)$ if $f=f_{1}+f_{2}$ with $f_{1} \in L^{p}\left(\mathbb{R}^{3}\right)$ and $f_{2} \in L^{q}\left(\mathbb{R}^{3}\right)$.

Lemma 3.2. Let $\Omega=\mathbb{R}^{3}$ and $\varepsilon, \mu \in W^{1, \infty}\left(\mathbb{R}^{3}\right)$ with $\varepsilon, \mu \geq \delta>0$ and $\partial_{i} \partial_{j} \varphi \in L^{3}\left(\mathbb{R}^{3}\right)+L^{\infty}\left(\mathbb{R}^{3}\right)$ for $\varphi \in\{\varepsilon, \mu\}$ and all $i, j \in\{1,2,3\}$. Then, it holds that $D\left(M_{0}^{2}\right) \hookrightarrow H^{2}\left(\mathbb{R}^{3}\right)^{6}$.

Proof. Let $w=(\mathbf{E}, \mathbf{H}) \in D\left(M_{0}^{2}\right)$. Since $\varepsilon$ and $\mu$ are Lipschitz and $\operatorname{div}(\varepsilon \mathbf{E})=0$, the function

$$
\operatorname{div} \mathbf{E}=\operatorname{div}\left(\varepsilon^{-1} \varepsilon \mathbf{E}\right)=\varepsilon^{-1} \operatorname{div}(\varepsilon \mathbf{E})+\nabla \varepsilon^{-1} \cdot \varepsilon \mathbf{E}=-\varepsilon^{-1} \nabla \varepsilon \cdot \mathbf{E}
$$

is contained in $L^{2}\left(\mathbb{R}^{3}\right)$, and analogously for $\mathbf{H}$. We compute

$$
\begin{aligned}
\operatorname{rot}\left(\frac{1}{\mu} \operatorname{rot} \mathbf{E}\right) & =\nabla \mu^{-1} \times \operatorname{rot} \mathbf{E}+\mu^{-1} \operatorname{rot} \operatorname{rot} \mathbf{E} \\
& =\nabla \mu^{-1} \times \operatorname{rot} \mathbf{E}+\mu^{-1}(-\Delta \mathbf{E}+\nabla \operatorname{div} \mathbf{E}) \\
& =-\mu^{-1} \Delta \mathbf{E}-\mu^{-2} \nabla \mu \times \operatorname{rot} \mathbf{E}-\mu^{-1} \nabla\left(\varepsilon^{-1} \nabla \varepsilon \cdot \mathbf{E}\right) .
\end{aligned}
$$

Note that the left hand side is equal to the first component of $-\varepsilon M^{2} w$ and thus its norm in $L^{2}\left(\mathbb{R}^{3}\right)^{3}$ is bounded by $c\left\|M^{2} w\right\|_{X}$. Moreover, $\|\operatorname{rot} \mathbf{E}\|_{L^{2}} \leq$ $c\|M w\|_{X} \leq c\left(\|w\|_{X}+\left\|M^{2} w\right\|_{X}\right)$. Hence, $\Delta \mathbf{E}$ belongs to $H^{-1}\left(\mathbb{R}^{3}\right)^{3} \supseteq$ $\nabla L^{2}\left(\mathbb{R}^{3}\right)$. Standard elliptic regularity results [19, Proposition 5.9.1] now imply that $\mathbf{E} \in H^{1}\left(\mathbb{R}^{3}\right)^{3}$ and $\|\mathbf{E}\|_{H^{1}} \leq c\left(\|w\|_{X}+\left\|M^{2} w\right\|_{X}\right)$. Sobolev's embedding theorem further yields $\mathbf{E} \in L^{6}\left(\mathbb{R}^{3}\right)^{3}$ so that the term $\nabla\left(\varepsilon^{-1} \nabla \varepsilon \cdot \mathbf{E}\right)$ is contained in $L^{2}\left(\mathbb{R}^{3}\right)^{3}$ by the assumptions on $\varepsilon$. From (16) we then infer that $\Delta \mathbf{E} \in L^{2}\left(\mathbb{R}^{3}\right)$ and $\|\Delta \mathbf{E}\|_{L^{2}} \leq c\left(\|w\|_{X}+\left\|M^{2} w\right\|_{X}\right)$. Again by elliptic regularity results [19, Proposition 5.9.1] it follows that $\mathbf{E} \in H^{2}\left(\mathbb{R}^{3}\right)^{3}$ and $\|\mathbf{E}\|_{H^{2}} \leq c\left(\|w\|_{X}+\left\|M^{2} w\right\|_{X}\right)$. $\mathbf{H}$ can be treated in the same way.

\subsection{Well-posedness and regularity on a Lipschitz domain}

We state and prove the basic facts for a general open set $\Omega \subset \mathbb{R}^{3}$ with a bounded Lipschitz boundary $\partial \Omega \neq \emptyset$ (and specialize to $\Omega=Q$ later). In this case the set $C^{\infty}(\bar{\Omega})^{3}$ is dense in $H(\operatorname{rot}, \Omega)$ and $H(\operatorname{div}, \Omega)$, see Theorems IX.1.1 
and IX.1.2 in [4]. We further need to explain the boundary conditions in (2). Let $R$ be the restriction map to $\partial \Omega$. Due to Theorem IX.1.2 of [4], the tangential trace $u \mapsto R u \times \nu$ (initially defined on $C^{\infty}(\bar{\Omega})^{3}$ ) extends to a bounded linear map from $H$ (rot) to $H^{-1 / 2}(\partial \Omega)^{3}$, which we still denote by $u \times \nu$ for simplicity. Moreover, we have the integration by parts formula

$$
\int_{\Omega} u \cdot \operatorname{rot} \varphi \mathrm{d} x=\int_{\Omega} \varphi \cdot \operatorname{rot} u \mathrm{~d} x+\langle u \times \nu, \varphi\rangle_{\partial \Omega} \quad \forall u \in H(\operatorname{rot}), \varphi \in H^{1}(\Omega)^{3},
$$

see (1.17) in Section IX.1 of [4]. Here the brackets designate the duality pairing between $H^{-1 / 2}(\partial \Omega)^{3}$ and $H^{1 / 2}(\partial \Omega)^{3}$ (and also between $H^{-1 / 2}(\partial \Omega$ ) and $\left.H^{1 / 2}(\partial \Omega)\right)$. We remark that the trace operator $\gamma$ maps $H^{1}(\Omega)$ onto $H^{1 / 2}(\partial \Omega)$ and that we usually write $\varphi$ instead of $\gamma \varphi$.

Similarly, the normal trace $v \mapsto R v \cdot \nu$ (defined on $C^{\infty}(\bar{\Omega})^{3}$ ) extends to a bounded linear map from $H(\operatorname{div})$ to $H^{-1 / 2}(\partial \Omega)$, denoted by $v \mapsto v \cdot \nu$. It also holds

$$
\int_{\Omega} v \cdot \nabla \psi \mathrm{d} x=-\int_{\Omega} \psi \operatorname{div} v \mathrm{~d} x+\langle v \cdot \nu, \psi\rangle_{\partial \Omega} \quad v \in H(\operatorname{div}), \psi \in H^{1}(\Omega),
$$

see Theorem IX.1.1 in [4]. We further need the closed subspace

$$
H_{0}(\operatorname{rot})=H_{0}(\operatorname{rot}, \Omega)=\{u \in H(\operatorname{rot}, \Omega) \mid u \times \nu=0 \text { on } \partial \Omega\}
$$

of $H(\operatorname{rot}, \Omega)$. By approximation, one can extend (17) to

$$
\int_{\Omega} u \cdot \operatorname{rot} \varphi \mathrm{d} x=\int_{\Omega} \varphi \cdot \operatorname{rot} u \mathrm{~d} x \quad \forall \varphi \in H(\operatorname{rot}), u \in H_{0}(\operatorname{rot}) .
$$

Test functions are dense in $H_{0}$ (rot) with respect to the norm in $H$ (rot), see Theorem IX.1.2 of [4]. The above traces of functions in $H$ (rot) and $H$ (div) are only distributions, in general, and thus a bit tricky. We add two technical remarks in this context which are needed below.

Remark 3.3. Traces like $\mu \mathbf{H} \cdot \nu=0$ as in (2) are defined for the product $\mu \mathbf{H} \in H$ (div). The product could be misleading here, as we do not claim that $\mu$ or $\mathbf{H}$ have a trace without further assumptions. However, if $\mu \in W^{1, \infty}(\Omega)$, $\mathbf{H} \in L^{2}(\Omega)^{3}$ and $\operatorname{div}(\mu \mathbf{H})=0$, then we derive $\mathbf{H} \in H(\operatorname{div})$ as in (14) so that the trace $\nu \cdot \mathbf{H}$ exists in $H^{-1 / 2}(\partial \Omega)$. To determine the trace, we take $\varphi \in H^{1}(\Omega)$ and set $\psi:=\mu^{-1} \varphi \in H^{1}(\Omega)$. Formula (18) yields

$$
\begin{aligned}
\langle\mathbf{H} \cdot \nu, \varphi\rangle_{\partial \Omega} & =\langle\mathbf{H} \cdot \nu, \mu \psi\rangle_{\partial \Omega}=\int_{\Omega}(\mu \psi \operatorname{div} \mathbf{H}+\nabla(\mu \psi) \cdot \mathbf{H}) \mathrm{d} x \\
& =\int_{\Omega}(\psi \operatorname{div}(\mu \mathbf{H})+\nabla \psi \cdot \mu \mathbf{H}) \mathrm{d} x=\langle\mu \mathbf{H} \cdot \nu, \psi\rangle_{\partial \Omega} .
\end{aligned}
$$

For $\mu \in W^{1, \infty}(\Omega)$, the boundary condition $\mu \mathbf{H} \cdot \nu=0$ is thus equivalent to $\mathbf{H} \cdot \nu=0$. In a similar way, for $\mathbf{H} \in H^{1}(\Omega)^{3}$ and $\mu \in W^{1, \infty}(\Omega)$ one shows that the trace of $\mu \mathbf{H}$ is the product of the traces of $\mu$ and $\mathbf{H}$, where all traces are functions. 
Remark 3.4. One can restrict the traces in $H$ (rot) and $H$ (div) to relatively open subsets $\Gamma_{0}$ of $\partial \Omega$. To this aim, let $\Gamma_{0}, \Gamma_{1} \subset \partial \Omega$ be disjoint and relatively open with $\overline{\Gamma_{0}} \cup \overline{\Gamma_{1}}=\partial \Omega$ such that $\partial \Gamma_{0}$ and $\partial \Gamma_{1}$ have surface measure 0 in $\partial \Omega$. Let $H_{\Gamma_{1}}^{1}(\Omega)^{3}$ be the subspace of functions $\varphi \in H^{1}(\Omega)^{3}$ whose traces vanish on $\Gamma_{1}$ (as an element of $\left.L^{2}(\partial \Omega)^{3}\right)$. The restriction $\phi_{\mid \Gamma_{0}}$ of a functional $\phi \in H^{-\frac{1}{2}}(\partial \Omega)^{3}$ to $\Gamma_{0}$ is defined as the restriction of $\phi$ to $H_{\Gamma_{1}}^{1}(\Omega)^{3}$. We also note that if $\phi_{\mid \Gamma_{0}}$ has a continuous extension to $L^{2}\left(\Gamma_{0}\right)^{3}$, then this extension is uniquely determined since $\gamma H_{\Gamma_{1}}^{1}(\Omega)^{3}$ is dense in $L^{2}\left(\Gamma_{0}\right)^{3}$ (and a subspace of $\left.H^{\frac{1}{2}}\left(\Gamma_{0}\right)^{3}\right)$, see Remarks 13.6.13 and 13.6.14 in [23].

For the investigation of (2), we use the state spaces $X=L^{2}(\Omega)^{6}$ and

$$
X_{0}=\left\{(\mathbf{E}, \mathbf{H}) \in L^{2}(\Omega)^{6} \mid \operatorname{div}(\varepsilon \mathbf{E})=\operatorname{div}(\mu \mathbf{H})=0, \mu \mathbf{H} \cdot \nu=0 \text { on } \partial \Omega\right\}
$$

with the scalar product given by (9). The subspace $X_{0}$ is closed in $X$ due to the closedness of div and the continuity of the normal trace. The Maxwell operator is now defined by

$$
M=\left(\begin{array}{cc}
0 & \frac{1}{\varepsilon} \operatorname{rot} \\
-\frac{1}{\mu} \operatorname{rot} & 0
\end{array}\right), \quad D(M)=D_{\Omega}(M)=H_{0}(\operatorname{rot}, \Omega) \times H(\operatorname{rot}, \Omega)
$$

in $X$. In view of (2), we mainly work with the restriction $M_{0}$ of $M$ to the domain

$$
D\left(M_{0}\right)=D_{\Omega}\left(M_{0}\right)=D_{\Omega}(M) \cap X_{0} .
$$

We see in the next result that $M$ maps $D(M)$ into $X_{0}$ and will thus consider $M_{0}$ as an operator in $X_{0}$.

Proposition 3.5. Let $\Omega \subset \mathbb{R}^{3}$ be open with a bounded Lipschitz boundary $\partial \Omega \neq \emptyset$ and let $\varepsilon, \mu \in L^{\infty}(\Omega)$ satisfy $\varepsilon, \mu \geq \delta>0$ for a constant $\delta>0$. Then the Maxwell operators $M$ and $M_{0}$ are skew-adjoint on $X$ and $X_{0}$, and thus generate unitary $C_{0}$-groups $T(t)=e^{t M}$ on $X$ and $T_{0}(t)=e^{t M_{0}}$ on $X_{0}$ for $t \in \mathbb{R}$, respectively. Therefore, for each $\left(\mathbf{E}^{0}, \mathbf{H}^{0}\right) \in D\left(M_{0}\right)$ we have a unique solution $(\mathbf{E}, \mathbf{H}) \in C^{1}\left(\mathbb{R} ; X_{0}\right) \cap C\left(\mathbb{R} ; D\left(M_{0}\right)\right)$ of $(2)$.

Moreover, $M$ maps $D(M)$ into $X_{0}$. Hence, $D\left(M_{0}^{j}\right)=D\left(M^{j}\right) \cap X_{0}$ and the operators $T_{0}(t)$ and $\left(\lambda I-M_{0}\right)^{-1}$ are the restrictions of $T(t)$ and $(\lambda I-M)^{-1}$ to $X_{0}$, for all $j \in \mathbb{N}, t \in \mathbb{R}$, and $\lambda \in \mathbb{R} \backslash\{0\}$.

Proof. We first show that $M$ maps $D(M)$ into $X_{0}$. In fact, the divergence conditions follow from div rot $=0$. Moreover, $\operatorname{rot} \mathbf{E}$ thus possesses a normal trace if $(\mathbf{E}, \mathbf{H}) \in D(M)$. Let $\varphi \in H^{2}(\Omega)$. The equations (18) and (17) then yield

$$
\begin{aligned}
\langle\nu \cdot \operatorname{rot} \mathbf{E}, \varphi\rangle_{\partial \Omega} & =-\int_{\Omega} \varphi \operatorname{div} \operatorname{rot} \mathbf{E} \mathrm{d} x+\langle\nu \cdot \operatorname{rot} \mathbf{E}, \varphi\rangle_{\partial \Omega}=\int_{\Omega} \operatorname{rot} \mathbf{E} \cdot \nabla \varphi \mathrm{d} x \\
& =\int_{\Omega} \mathbf{E} \cdot \operatorname{rot} \nabla \varphi \mathrm{d} x-\langle\mathbf{E} \times \nu, \nabla \varphi\rangle_{\partial \Omega}=0,
\end{aligned}
$$


since $\operatorname{rot} \nabla=0$ and $\mathbf{E} \in H_{0}$ (rot). By approximation, we deduce that $\langle\nu$. $\operatorname{rot} \mathbf{E}, \varphi\rangle_{\partial \Omega}=0$ for all $\varphi \in H^{1}(\Omega)$, and hence $\nu \cdot \mu \frac{1}{\mu} \operatorname{rot} \mathbf{E}=\nu \cdot \operatorname{rot} \mathbf{E}=0$ as asserted.

The operators $M$ and $M_{0}$ are closed in $X$ and $X_{0}$, respectively, because of the closedness of $X_{0}$ and rot in $X$ and the continuity of the tangential trace.

As in the proof of Proposition 3.1, one derives the skew-symmetry of $M$ and $M_{0}$ now using (19). To show the range condition, one again employs the symmetric form $a(\cdot, \cdot)$ from (13) (with $\Omega$ instead of $\mathbb{R}^{3}$ ) which is defined on $H_{0}(\operatorname{rot}, \Omega)$ this time. The remaining assertions then follow as in the proof of Proposition 3.1.

We now come back to the special case $Q=\left(a_{1}^{-}, a_{1}^{+}\right) \times\left(a_{2}^{-}, a_{2}^{+}\right) \times\left(a_{3}^{-}, a_{3}^{+}\right)$. To transfer Lemma 3.2 to the present setting, we have to work much harder because of the boundary conditions. We need an auxiliary result ensuring $H^{2}$ regularity of the Laplacian on $Q$ with mixed boundary conditions. It is surely known to experts, but since we could not detect a proof in the literature we present it in the appendix.

We employ the isometric isomorphisms

$$
\begin{aligned}
D_{1}=\left\{v \in L^{2}(Q) \mid \partial_{1} v \in L^{2}(Q)\right\} & \cong L^{2}\left(\left(a_{2}^{-}, a_{2}^{+}\right) \times\left(a_{3}^{-}, a_{3}^{+}\right) ; H^{1}\left(a_{1}^{-}, a_{1}^{+}\right)\right) \\
& \cong H^{1}\left(\left(a_{1}^{-}, a_{1}^{+}\right) ; L^{2}\left(\left(a_{2}^{-}, a_{2}^{+}\right) \times\left(a_{3}^{-}, a_{3}^{+}\right)\right)\right),
\end{aligned}
$$

and their analogues for $\partial_{2}$ and $\partial_{3}$ which follow easily from the corresponding isomorphisms with $H^{1}$ replaced by $L^{2}$. As a result, a function in $D_{1}$ has traces to $\Gamma_{1}^{ \pm}$that belong to $L^{2}\left(\left(a_{2}^{-}, a_{2}^{+}\right) \times\left(a_{3}^{-}, a_{3}^{+}\right)\right)$. The space $H_{\Gamma}^{1}(Q)$ is defined as in Remark 3.4.

Lemma 3.6. Let $\Gamma$ be a union of some of the six open faces of $Q, \Gamma^{\prime}$ be the union of the remaining open faces. Let $f \in L^{2}(Q)$. Then there is a unique function $v \in H_{\Gamma}^{1}(Q)$ such that

$$
\int_{Q} v \varphi \mathrm{d} x+\int_{Q} \nabla v \cdot \nabla \varphi \mathrm{d} x=\int_{Q} f \varphi \mathrm{d} x \quad \text { for all } \varphi \in H_{\Gamma}^{1}(Q) .
$$

Morever, the function $v$ belongs to $D:=\left\{v \in H^{2}(Q) \cap H_{\Gamma}^{1}(Q) \mid \partial_{\nu} v=0\right.$ on $\left.\Gamma^{\prime}\right\}$ and $v-\Delta v=f$. Finally, the $H^{2}{ }^{-}$norm and the graph norm of $\Delta$ are equivalent on $D$.

The following results about regularity and boundary traces for $(\mathbf{E}, \mathbf{H}) \in$ $D\left(M_{0}^{2}\right)$ are crucial for our error analysis. As in Lemma 3.2 we need some smoothness of the coefficients. The regularity of the fields seem also to follow if one applies Theorem 4.8 of [3] to $\varepsilon \mathbf{E}$ and $\mu \mathbf{H}$ (cf. Paragraph 4.4 .2 in [3]). However, the results in [3] are obtained in a framework of an elaborate study of singularities of time harmonic Maxwell equations in general polyhedral domains. In our opinion it is very useful to include a rather short, direct proof for the non-singular situation of a cuboid, which is given in the appendix. 
Lemma 3.7. Let $\varepsilon, \mu \in W^{1, \infty}(Q)$ with $\varepsilon, \mu \geq \delta>0$ and $\partial_{i} \partial_{j} \varphi \in L^{3}(Q)$ for $\varphi \in\{\varepsilon, \mu\}$ and for all $i, j \in\{1,2,3\}$. It then holds $D\left(M_{0}^{2}\right) \hookrightarrow H^{2}(Q)^{6}$ and $(\mathbf{E}, \mathbf{H}) \in D\left(M_{0}^{2}\right)$ has the traces

$$
\begin{aligned}
& \text { on } \Gamma_{1}^{ \pm}: E_{2}=E_{3}=0, \quad \partial_{2} E_{2}=\partial_{3} E_{2}=\partial_{2} E_{3}=\partial_{3} E_{3}=0, \\
& \text { on } \Gamma_{2}^{ \pm}: E_{1}=E_{3}=0, \quad \partial_{1} E_{1}=\partial_{3} E_{1}=\partial_{1} E_{3}=\partial_{3} E_{3}=0, \\
& \text { on } \Gamma_{3}^{ \pm}: E_{1}=E_{2}=0, \quad \partial_{1} E_{1}=\partial_{2} E_{1}=\partial_{1} E_{2}=\partial_{2} E_{2}=0, \\
& \text { on } \Gamma_{1}^{ \pm}: H_{1}=0, \quad \partial_{2} H_{1}=\partial_{3} H_{1}=0, \\
& \text { on } \Gamma_{2}^{ \pm}: H_{2}=0, \quad \partial_{1} H_{2}=\partial_{3} H_{2}=0, \\
& \text { on } \Gamma_{3}^{ \pm}: H_{3}=0, \quad \partial_{1} H_{3}=\partial_{2} H_{3}=0 .
\end{aligned}
$$

\section{Error analysis}

For the analysis of the splitting scheme (4), we define the operators

$$
\Lambda_{j}(\tau) w=\frac{1}{\tau^{j}(j-1) !} \int_{0}^{\tau}(\tau-s)^{j-1} T_{0}(s) w \mathrm{~d} s
$$

for $j \in \mathbb{N}, \tau>0$ and $w \in X_{0}$; cf. [13]. It can be checked that $\left\|\Lambda_{j}(\tau)\right\|_{X_{0}} \leq$ $1 /(j !) \leq 1$. Setting $\Lambda_{0}(\tau)=T_{0}(\tau)$, one easily shows that

$$
\tau M_{0} \Lambda_{j+1}(\tau) w=\Lambda_{j}(\tau) w-\frac{1}{j !} w
$$

for all $w \in D\left(M_{0}\right), \tau>0$ and $j \in \mathbb{N}_{0}$. In particular,

$$
\Lambda_{0}=I+\tau M_{0} \Lambda_{1}=I+\tau M_{0}+\tau^{2} M_{0}^{2} \Lambda_{2}=I+\tau M_{0}+\frac{1}{2} \tau^{2} M_{0}^{2}+\tau^{3} M_{0}^{3} \Lambda_{3}
$$

on $D\left(M_{0}^{3}\right)$, with $\Lambda_{j}:=\Lambda_{j}(\tau)$.

\subsection{Splitting for Maxwell's equations on $\mathbb{R}^{3}$}

The Peaceman-Rachford scheme (4) involves resolvents and Cayley transforms of $\tau A$ and $\tau B$. For the stability of the scheme, these operators should be contractive which requires the dissipativity of $A$ and $B$. Actually, we can prove even their skew-adjointness without assuming extra regularity for $\varepsilon$ and $\mu$. We point out that $A$ and $B$ act on $X$ and not on $X_{0}$.

Lemma 4.1. Let $\varepsilon, \mu \in L^{\infty}\left(\mathbb{R}^{3}\right)$ with $\varepsilon, \mu \geq \delta>0$. Then $A$ and $B$ are skewadjoint in $X$, and hence the operators $(I-\tau A)^{-1},(I-\tau B)^{-1},(I+\tau A)(I-$ $\tau A)^{-1}$ and $(I+\tau B)(I-\tau B)^{-1}$ are contractive in $X$ for each $\tau>0$.

Proof. We only consider $A$ since the proof for $B$ is analogous. We will show that $A$ is skew-symmetric and that $I \pm A$ has dense range. Clearly, $A$ is closed. The skew-adjointness of $A$ then follows, which implies the other properties. Let $(u, v),(\varphi, \psi) \in D(A)$. Integrating by parts, we deduce

$$
(A(u, v) \mid(\varphi, \psi))_{X}=\left(\varepsilon^{-1} C_{1} v \mid \varphi\right)_{\varepsilon}+\left(\mu^{-1} C_{2} u \mid \psi\right)_{\mu}
$$




$$
\begin{aligned}
& =\int_{\mathbb{R}^{3}}\left(\left(\partial_{2} v_{3} \varphi_{1}+\partial_{3} v_{1} \varphi_{2}+\partial_{1} v_{2} \varphi_{3}\right)+\left(\partial_{3} u_{2} \psi_{1}+\partial_{1} u_{3} \psi_{2}+\partial_{2} u_{1} \psi_{3}\right)\right) \mathrm{d} x \\
& =-\int_{\mathbb{R}^{3}}\left(v_{3} \partial_{2} \varphi_{1}+v_{1} \partial_{3} \varphi_{2}+v_{2} \partial_{1} \varphi_{3}+u_{2} \partial_{3} \psi_{1}+u_{3} \partial_{1} \psi_{2}+u_{1} \partial_{2} \psi_{3}\right) \mathrm{d} x \\
& =-\int_{\mathbb{R}^{3}}\left(\varepsilon u \cdot \frac{1}{\varepsilon} C_{1} \psi+\mu v \cdot \frac{1}{\mu} C_{2} \varphi\right) \mathrm{d} x=-((u, v) \mid A(\varphi, \psi))_{X} .
\end{aligned}
$$

To check the range condition, we take $(\varphi, \psi) \in X$ such that $\partial_{2} \psi_{3}, \partial_{3} \psi_{1}$ and $\partial_{1} \psi_{2}$ belong to $L^{2}(Q)^{3}$. We then look for $(\mathbf{E}, \mathbf{H}) \in D(A)$ such that $(\mathbf{E}, \mathbf{H}) \pm$ $A(\mathbf{E}, \mathbf{H})=(\varphi, \psi)$. Reordering the lines, we write these equations as

$$
\begin{array}{ll}
E_{1} \pm \frac{1}{\varepsilon} \partial_{2} H_{3}=\varphi_{1}, & H_{3} \pm \frac{1}{\mu} \partial_{2} E_{1}=\psi_{3}, \\
E_{2} \pm \frac{1}{\varepsilon} \partial_{3} H_{1}=\varphi_{2}, & H_{1} \pm \frac{1}{\mu} \partial_{3} E_{2}=\psi_{1}, \\
E_{3} \pm \frac{1}{\varepsilon} \partial_{1} H_{2}=\varphi_{3}, & H_{2} \pm \frac{1}{\mu} \partial_{1} E_{3}=\psi_{2} .
\end{array}
$$

Formally, we insert the equations in the second column in the corresponding ones in the first column and multiply by $\varepsilon$, arriving at

$$
\begin{aligned}
& \varepsilon E_{1}-\partial_{2}\left(\frac{1}{\mu} \partial_{2} E_{1}\right)=\varepsilon \varphi_{1} \mp \partial_{2} \psi_{3}=: f_{1} \in L^{2}(Q), \\
& \varepsilon E_{2}-\partial_{3}\left(\frac{1}{\mu} \partial_{3} E_{2}\right)=\varepsilon \varphi_{2} \mp \partial_{3} \psi_{1}=: f_{2} \in L^{2}(Q), \\
& \varepsilon E_{3}-\partial_{1}\left(\frac{1}{\mu} \partial_{1} E_{3}\right)=\varepsilon \varphi_{3} \mp \partial_{1} \psi_{2}=: f_{3} \in L^{2}(Q) .
\end{aligned}
$$

We now start to solve these equations. To this aim, we introduce the operator $D_{j}=\partial_{j} \frac{1}{\mu} \partial_{j}$ with domain

$$
D\left(D_{j}\right)=\left\{u \in L^{2}\left(\mathbb{R}^{3}\right)^{3} \mid \partial_{j} u, D_{j} u \in L^{2}\left(\mathbb{R}^{3}\right)^{3}\right\}
$$

with $j=1,2,3$. Using Lax-Milgram, one obtains functions $E_{k(j)} \in D\left(D_{j}\right)$ such that $\varepsilon E_{k(j)}-D_{k(j)} E_{k(j)}=f_{k(j)}$, with $k(1)=3, k(2)=1$ and $k(3)=2$. We then define

$$
H_{1}=\mp \frac{1}{\mu} \partial_{3} E_{2}+\psi_{1}, \quad H_{2}=\mp \frac{1}{\mu} \partial_{1} E_{3}+\psi_{2}, \quad H_{3}=\mp \frac{1}{\mu} \partial_{2} E_{1}+\psi_{3} .
$$

Hence, $(\mathbf{E}, \mathbf{H})$ belongs to $D(A)$ and satisfies $(\mathbf{E}, \mathbf{H}) \pm A(\mathbf{E}, \mathbf{H})=(\varphi, \psi)$.

We now state our convergence result for the full space. We point out that the convergence estimate is of second order and that it is proportional to a 'third order norm' (the graph norm of $M_{0}^{3}$ ) of the initial value, cf. Section 4.4.

Theorem 4.2. Let $\varepsilon, \mu \in W^{1, \infty}\left(\mathbb{R}^{3}\right)$ with $\varepsilon, \mu \geq \delta>0$ and $\partial_{i} \partial_{j} \varepsilon, \partial_{i} \partial_{j} \mu \in$ $L^{3}\left(\mathbb{R}^{3}\right)+L^{\infty}\left(\mathbb{R}^{3}\right)$ for all $i, j \in\{1,2,3\}$. Then there is a constant $c>0$ such that the splitting operator $S_{\tau}$ defined in (4) satisfies

$$
\left\|S_{\tau}^{n} w-T_{0}(n \tau) w\right\|_{L^{2}} \leq c t_{\text {end }} \tau^{2}\left(\|w\|_{L^{2}}+\left\|M_{0}^{3} w\right\|_{L^{2}}\right)
$$

for all $w=(\mathbf{E}, \mathbf{H}) \in D\left(M^{3}\right) \cap X_{0}=D\left(M_{0}^{3}\right), n \in \mathbb{N}, \tau>0$ and $t_{\text {end }}>0$ with $n \tau \leq t_{\text {end }}$. 
Proof. Our proof is based on a formula for the difference $S_{\tau}^{n}-T_{0}(\tau n)$ which was established in the proof of Theorem 3.2 of [13] for the case that $A, B$ and $M_{0}$ act on the same spaces. We fix $\tau>0$ and $w \in D\left(M^{3}\right) \cap X_{0}$. Then $M_{0}^{k} \Lambda_{j}(\tau) w$ belongs to $D\left(M_{0}^{3-k}\right) \subset D(A B) \cap D(A)$ for $k=0,1$ and $j \in \mathbb{N}_{0}$ by Lemma 3.2 and the definition $(22)$ of $\Lambda_{j}(\tau)$. We set $R_{A}=\left(I-\frac{\tau}{2} A\right)^{-1}$ and $R_{B}=\left(I-\frac{\tau}{2} B\right)^{-1}$. Recall that $A w+B w=M_{0} w$. Using the formulas (23) for $\Lambda_{0}(\tau)=T_{0}(\tau)$, we compute

$$
\begin{aligned}
S_{\tau} w-T_{0}(\tau) w= & R_{B} R_{A}\left[\left(I+\frac{\tau}{2} A\right)\left(I+\frac{\tau}{2} B\right)-\left(I-\frac{\tau}{2} A\right)\left(I-\frac{\tau}{2} B\right) T_{0}(\tau)\right] w \\
= & R_{B} R_{A}\left[I+\frac{\tau}{2} M_{0}+\frac{\tau^{2}}{4} A B-\left(I-\frac{\tau}{2} M_{0}+\frac{\tau^{2}}{4} A B\right) \Lambda_{0}(\tau)\right] w \\
= & R_{B} R_{A}\left[I-\Lambda_{0}(\tau)+\frac{\tau}{2} M_{0}\left(I+\Lambda_{0}(\tau)\right)+\frac{\tau^{2}}{4} A B\left(I-\Lambda_{0}(\tau)\right)\right] w \\
= & R_{B} R_{A}\left[-\tau M_{0}-\frac{\tau^{2}}{2} M_{0}^{2}-\tau^{3} M_{0}^{3} \Lambda_{3}(\tau)+\frac{\tau}{2} M_{0}\left(2+\tau M_{0}\right.\right. \\
& \left.\left.\quad+\tau^{2} M_{0}^{2} \Lambda_{2}(\tau)\right)-\frac{\tau^{3}}{4} A B M_{0} \Lambda_{1}(\tau)\right] w \\
= & \tau^{3} R_{B} R_{A}\left[\left(\frac{1}{2} \Lambda_{2}(\tau)-\Lambda_{3}(\tau)\right) M_{0}^{3}-\frac{1}{4} A B M_{0} \Lambda_{1}(\tau)\right] w .
\end{aligned}
$$

A telescoping sum then leads to

$$
\begin{aligned}
S_{\tau}^{n} w- & T_{0}(n \tau) w=\sum_{j=0}^{n-1} S_{\tau}^{n-j-1}\left(S_{\tau}-T_{0}(\tau)\right) T_{0}(j \tau) w \\
= & \tau^{3} \sum_{j=0}^{n-1} S_{\tau}^{n-j-1}\left(I-\frac{\tau}{2} B\right)^{-1}\left(I-\frac{\tau}{2} A\right)^{-1}\left[\frac{1}{2} \Lambda_{2}(\tau)-\Lambda_{3}(\tau)\right] T_{0}(j \tau) M_{0}^{3} w \\
& -\frac{\tau^{3}}{4} \sum_{j=0}^{n-1} S_{\tau}^{n-j-1}\left(I-\frac{\tau}{2} B\right)^{-1}\left(I-\frac{\tau}{2} A\right)^{-1} A B\left(I-M_{0}\right)^{-2} \Lambda_{1}(\tau) T_{0}(j \tau) w^{\prime}
\end{aligned}
$$

with $w^{\prime}=\left(I-M_{0}\right)^{2} M_{0} w$. Lemmas 3.2 and 4.1 and the contractivity of $\Lambda_{j}(\tau)$ and $T_{0}(t)$ now imply the assertion.

\subsection{Splitting for Maxwell's equations on the cuboid $Q$}

We first note that the boundary conditions in $D_{Q}(A)$ and $D_{Q}(B)$ are well defined in view of the discussion before Lemma 3.6. Moreover, the traces appearing in the definition of $D_{Q}(A)$ and $D_{Q}(B)$ are continuous from the respective domain into the $L^{2}$ space on the relevant face due to this discussion. As a result, $A$ and $B$ are closed in $X$. Again we can show their skew-adjointness.

Lemma 4.3. Let $\varepsilon, \mu \in L^{\infty}(Q)$ with $\varepsilon, \mu \geq \delta>0$. Then $A$ and $B$ are skewadjoint in $X$, and hence the operators $(I-\tau A)^{-1},(I-\tau B)^{-1},(I+\tau A)(I-$ $\tau A)^{-1}$ and $(I+\tau B)(I-\tau B)^{-1}$ are contractive in $X$ for each $\tau>0$. 
Proof. The proof is almost identical to that of Lemma 4.1. One can repeat the calculations in (24) on the spatial domain $Q$ since all boundary terms in the integration by parts vanish thanks to the boundary conditions in $D_{Q}(A)$. Hence, $A$ is skew-symmetric. In the proof of the range condition we only have to change the domain of $D_{j}$ into

$$
D\left(D_{j}\right)=\left\{u \in L^{2}(Q)^{3} \mid \partial_{j} u, D_{j} u \in L^{2}(Q)^{3}, u=0 \text { on } \Gamma_{j}^{ \pm}\right\} .
$$

One then finishes the proof as in Lemma 4.1

Since both $A B$ and $M_{0}$ are of second order, one may expect that $A B(I-$ $\left.M_{0}\right)^{-2}$ is bounded. This crucial fact directly follows from Lemma 3.7 which gives the needed $H^{2}$ regularity and boundary conditions for $w \in D\left(M_{0}^{2}\right)$.

Proposition 4.4. Let $\varepsilon, \mu \in W^{1, \infty}(Q)$ with $\varepsilon, \mu \geq \delta>0$ and $\partial_{i} \partial_{j} \varphi \in L^{3}(Q)$ for $\varphi \in\{\varepsilon, \mu\}$ and all $i, j \in\{1,2,3\}$. Then $D\left(M_{0}^{2}\right)=D\left(M^{2}\right) \cap X_{0} \hookrightarrow H^{2}(Q)^{6} \cap$ $D(A B) \cap D(A)$ and $A B\left(I-M_{0}\right)^{-2}: X_{0} \rightarrow X$ is bounded.

Using the above proposition and $D(A) \cap D(B) \subseteq D(M)$, one can now establish our main convergence result on $Q$ exactly as for $\mathbb{R}^{3}$.

Theorem 4.5. Let $\varepsilon, \mu \in W^{1, \infty}(Q)$ with $\varepsilon, \mu \geq \delta>0$ and $\partial_{i} \partial_{j} \varepsilon, \partial_{i} \partial_{j} \mu \in$ $L^{3}(Q)$ for all $i, j \in\{1,2,3\}$. Then there is a constant $c>0$ such that the splitting operator $S_{\tau}$ defined in (4) satisfies

$$
\left\|S_{\tau}^{n} w-T_{0}(n \tau) w\right\|_{L^{2}} \leq c t_{\mathrm{end}} \tau^{2}\left(\|w\|_{L^{2}}+\left\|M_{0}^{3} w\right\|_{L^{2}}\right.
$$

for all $w=(\mathbf{E}, \mathbf{H}) \in D\left(M^{3}\right) \cap X_{0}=D\left(M_{0}^{3}\right), n \in \mathbb{N}, \tau>0$ and $t_{\mathrm{end}}>0$ with $n \tau \leq t_{\text {end }}$

4.3 Equivalence of the efficient reformulation of the method on the cuboid $Q$

In order to extend the efficient scheme from Section 2.3 to the case with boundary conditions, we use weak formulations of (6) and (7). We introduce the relevant test function spaces

$$
\begin{aligned}
& Y_{1}=\left\{u \in L^{2}(Q)^{3} \mid \partial_{3} u_{1}, \partial_{1} u_{2}, \partial_{2} u_{3} \in L^{2}(Q)^{3} ;\right. \\
& \left.u_{1}=0 \text { on } \Gamma_{3}^{ \pm}, u_{2}=0 \text { on } \Gamma_{1}^{ \pm}, u_{3}=0 \text { on } \Gamma_{2}^{ \pm}\right\}, \\
& Y_{2}=\left\{u \in L^{2}(Q)^{3} \mid \partial_{2} u_{1}, \partial_{3} u_{2}, \partial_{1} u_{3} \in L^{2}(Q)^{3} ;\right. \\
& \left.u_{1}=0 \text { on } \Gamma_{2}^{ \pm}, u_{2}=0 \text { on } \Gamma_{3}^{ \pm}, u_{3}=0 \text { on } \Gamma_{1}^{ \pm}\right\} .
\end{aligned}
$$

Observe that for $(u, \tilde{u}) \in D(A),(v, \tilde{v}) \in D(B)$ and $\varphi \in Y_{j}$, we have $u \in Y_{2}$, $v \in Y_{1}$ and $C_{j} \varphi \in L^{2}(Q)^{3}$. Integration by parts shows that

$$
\int_{Q} C_{2} u \cdot \psi \mathrm{d} x=-\int_{Q} u \cdot C_{1} \psi \mathrm{d} x, \quad \int_{Q} C_{1} v \cdot \varphi \mathrm{d} x=-\int_{Q} v \cdot C_{2} \varphi \mathrm{d} x
$$

for all $u \in Y_{2}, v \in Y_{1}$ and $\varphi, \psi \in L^{2}(Q)^{3}$ with $C_{1} \psi, C_{2} \varphi \in L^{2}(Q)^{3}$. In the next result, we use the weak versions of the differential operators in (8). 
Proposition 4.6. Let $\varepsilon, \mu \in W^{1, \infty}(Q)$ with $\varepsilon, \mu \geq \delta>0$ and $\partial_{i} \partial_{j} \varepsilon, \partial_{i} \partial_{j} \mu \in$ $L^{3}(Q)$ for all $i, j \in\{1,2,3\}$, and let $\left(\mathbf{E}^{0}, \mathbf{H}^{0}\right) \in D\left(M^{3}\right) \cap X_{0}$. We consider the approximations given by $(5)$. Then, $(u, v)=\left(\mathbf{E}^{n+\frac{1}{2}}, \mathbf{H}^{n+\frac{1}{2}}\right)$ is the unique solution in $D(A)$ of the decoupled system

$$
\begin{aligned}
(u \mid \varphi)_{\varepsilon}+\frac{\tau^{2}}{4}\left(\frac{1}{\mu} C_{2} u \mid \frac{1}{\varepsilon} C_{2} \varphi\right)_{\varepsilon}= & \left(\mathbf{E}^{n} \mid \varphi\right)_{\varepsilon}-\frac{\tau}{2}\left(\mathbf{H}^{n} \mid \frac{1}{\varepsilon} C_{2} \varphi\right)_{\varepsilon}-\frac{\tau}{2}\left(\frac{1}{\mu} C_{2} \mathbf{H}^{n} \mid \varphi\right)_{\mu} \\
& +\frac{\tau^{2}}{4}\left(\frac{1}{\mu} C_{1} \mathbf{E}^{n} \mid \frac{1}{\varepsilon} C_{2} \varphi\right)_{\varepsilon} \quad \forall \varphi \in Y_{2} \\
v= & H^{n}-\frac{\tau}{2 \mu} C_{1} \mathbf{E}^{n}+\frac{\tau}{2 \mu} C_{2} u .
\end{aligned}
$$

Moreover, $(u, v)=\left(\mathbf{E}^{n+1}, \mathbf{H}^{n+1}\right)$ is the unique solution in $D(B)$ of the decoupled system

$$
\begin{aligned}
(u \mid \psi)_{\varepsilon}+\frac{\tau^{2}}{4}\left(\frac{1}{\mu} C_{1} u \mid \frac{1}{\varepsilon} C_{1} \psi\right)_{\varepsilon}= & \left(\mathbf{E}^{n+\frac{1}{2}} \mid \psi\right)_{\varepsilon}+\frac{\tau}{2}\left(\mathbf{H}^{n+\frac{1}{2}} \mid \frac{1}{\varepsilon} C_{1} \psi\right)_{\varepsilon}+\frac{\tau}{2}\left(\frac{1}{\mu} C_{1} \mathbf{H}^{n+\frac{1}{2}} \mid \psi\right)_{\mu} \\
& +\frac{\tau^{2}}{4}\left(\frac{1}{\mu} C_{2} \mathbf{E}^{n+\frac{1}{2}} \mid \frac{1}{\varepsilon} C_{1} \psi\right)_{\varepsilon} \quad \forall \psi \in Y_{1}, \\
v= & \mathbf{H}^{n+\frac{1}{2}}+\frac{\tau}{2 \mu} C_{2} \mathbf{E}^{n+\frac{1}{2}}-\frac{\tau}{2 \mu} C_{1} u .
\end{aligned}
$$

Proof. We focus on the first halfstep (27) since the second one can be treated in the same way. Let $(\varphi, \psi) \in D(A)$, i.e., $\varphi \in Y_{2}$ and $C_{1} \psi \in L^{2}(Q)^{3}$. First, a standard application of Lax-Milgram gives a solution $u \in Y_{2}$ of (27) for each $\left(\mathbf{E}^{n}, \mathbf{H}^{n}\right) \in D(B)$. We then define $v \in L^{2}(Q)^{3}$ by (28). Taking the $\varepsilon$-scalar product of (28) with $\frac{\tau}{2 \varepsilon} C_{2} \varphi$ and adding it to the equation for $u$, we deduce

$$
(u \mid \varphi)_{\varepsilon}+\frac{\tau}{2}\left(v \mid \frac{1}{\varepsilon} C_{2} \varphi\right)_{\varepsilon}=\left(\mathbf{E}^{n} \mid \varphi\right)_{\varepsilon}-\frac{\tau}{2}\left(\frac{1}{\mu} C_{2} \mathbf{H}^{n} \mid \varphi\right)_{\mu},
$$

which yields

$$
(u \mid \varphi)_{\varepsilon}+\frac{\tau}{2}\left(v \mid \frac{1}{\mu} C_{2} \varphi\right)_{\mu}=\left(\mathbf{E}^{n} \mid \varphi\right)_{\varepsilon}-\frac{\tau}{2}\left(\frac{1}{\varepsilon} C_{2} \mathbf{H}^{n} \mid \varphi\right)_{\varepsilon} .
$$

We further take the $\mu$-scalar product of (28) with $\psi$ and obtain

$$
\begin{aligned}
(v \mid \psi)_{\mu}-\frac{\tau}{2}\left(C_{2} u \mid \psi\right) & =\left(\mathbf{H}^{n} \mid \psi\right)_{\mu}-\frac{\tau}{2}\left(\frac{1}{\mu} C_{1} \mathbf{E}^{n} \mid \psi\right)_{\mu} \\
(v \mid \psi)_{\mu}+\frac{\tau}{2}\left(u \mid \frac{1}{\varepsilon} C_{1} \psi\right)_{\varepsilon} & =\left(\mathbf{H}^{n} \mid \psi\right)_{\mu}-\frac{\tau}{2}\left(\frac{1}{\mu} C_{1} \mathbf{E}^{n} \mid \psi\right)_{\mu}
\end{aligned}
$$

where we use (26). The sum of (31) and (32) can be written as

$$
\left((u, v) \mid\left(I+\frac{\tau}{2} A\right)(\varphi, \psi)\right)_{X}=\left(\left(I+\frac{\tau}{2} B\right)\left(\mathbf{E}^{n}, \mathbf{H}^{n}\right) \mid(\varphi, \psi)\right)_{X}
$$

for all $(\varphi, \psi) \in D(A)$. On the other hand, (5) and Lemma 4.3 imply that

$$
\left(\left(\mathbf{E}^{n+\frac{1}{2}}, \mathbf{H}^{n+\frac{1}{2}}\right) \mid\left(I+\frac{\tau}{2} A\right)(\varphi, \psi)\right)_{X}=\left(\left(I+\frac{\tau}{2} B\right)\left(\mathbf{E}^{n}, \mathbf{H}^{n}\right) \mid(\varphi, \psi)\right)_{X}
$$

holds for all $(\varphi, \psi) \in D(A)$. The difference $\left(\mathbf{E}^{n+\frac{1}{2}}-u, \mathbf{H}^{n+\frac{1}{2}}-v\right) \in X$ thus belongs to the kernel of $\left(I+\frac{\tau}{2} A\right)^{*}=\left(I-\frac{\tau}{2} A\right)$ which is trivial. Consequently, $\left(\mathbf{E}^{n+\frac{1}{2}}, \mathbf{H}^{n+\frac{1}{2}}\right) \in D(A)$ satisfies $(27)$. 
4.4 Numerical examples

In order to illustrate Theorem 4.5 we apply the numerical method (6)-(7) to two model problems. In both cases, we consider Maxwell's equations (2) on the unit cube $(0,1) \times(0,1) \times(0,1)$. For the spatial discretization the classical Yee grid (cf. [27] or Section 3.6 in [22]) with mesh width $h=1 / m$ is used $(m \in \mathbb{N})$. Hence, numerical approximations

$$
\begin{aligned}
E_{1}^{n}\left(i+\frac{1}{2}, j, k\right) & \approx \mathbf{E}_{1}\left(t_{n},\left(i+\frac{1}{2}\right) h, j h, k h\right), \\
E_{2}^{n}\left(i, j+\frac{1}{2}, k\right) & \approx \mathbf{E}_{2}\left(t_{n}, i h,\left(j+\frac{1}{2}\right) h, k h\right), \\
E_{3}^{n}\left(i, j, k+\frac{1}{2}\right) & \approx \mathbf{E}_{3}\left(t_{n}, i h, j h,\left(k+\frac{1}{2}\right) h\right), \\
H_{1}^{n}\left(i, j+\frac{1}{2}, k+\frac{1}{2}\right) & \approx \mathbf{H}_{1}\left(t_{n}, i h,\left(j+\frac{1}{2}\right) h,\left(k+\frac{1}{2}\right) h\right), \\
H_{2}^{n}\left(i+\frac{1}{2}, j, k+\frac{1}{2}\right) & \approx \mathbf{H}_{2}\left(t_{n},\left(i+\frac{1}{2}\right) h, j h,\left(k+\frac{1}{2}\right) h\right), \\
H_{3}^{n}\left(i+\frac{1}{2}, j+\frac{1}{2}, k\right) & \approx \mathbf{H}_{3}\left(t_{n},\left(i+\frac{1}{2}\right) h,\left(j+\frac{1}{2}\right) h, k h\right),
\end{aligned}
$$

are computed on six different staggered grids, and all partial derivatives are approximated by central finite differences, for example

$$
\begin{aligned}
\partial_{2} \mathbf{H}_{3}\left(t_{n},\left(i+\frac{1}{2}\right) h, j h, k h\right) & \approx \frac{H_{3}^{n}\left(i+\frac{1}{2}, j+\frac{1}{2}, k\right)-H_{3}^{n}\left(i+\frac{1}{2}, j-\frac{1}{2}, k\right)}{h} \\
\partial_{3} \mathbf{H}_{2}\left(t_{n},\left(i+\frac{1}{2}\right) h, j h, k h\right) & \approx \frac{H_{2}^{n}\left(i+\frac{1}{2}, j, k+\frac{1}{2}\right)-H_{2}^{n}\left(i+\frac{1}{2}, j, k-\frac{1}{2}\right)}{h}
\end{aligned}
$$

and so on. Note that $\partial_{3} \mathbf{H}_{2}$ and $\partial_{2} \mathbf{H}_{3}$ are not approximated on the same grid as $\mathbf{H}_{2}$ and $\mathbf{H}_{3}$, respectively, but on the same grid as $\mathbf{E}_{1}$. This makes sense because (1) or (2) imply that

$$
\partial_{t} \mathbf{E}_{1}=\varepsilon^{-1}\left(\partial_{2} \mathbf{H}_{3}-\partial_{3} \mathbf{H}_{2}\right)
$$

The other field components $\mathbf{E}_{2}, \mathbf{E}_{3}, \mathbf{H}_{1}, \mathbf{H}_{2}$, and $\mathbf{H}_{3}$ are treated similarly. The boundary conditions are implemented in a straightforward way: we simply let

$$
\begin{array}{ll}
E_{2}^{n}\left(i, j+\frac{1}{2}, k\right)=E_{3}^{n}\left(i, j, k+\frac{1}{2}\right)=0 & \text { for } i \in\{0, m\}, \\
E_{1}^{n}\left(i+\frac{1}{2}, j, k\right)=E_{3}^{n}\left(i, j, k+\frac{1}{2}\right)=0 & \text { for } j \in\{0, m\}, \\
E_{1}^{n}\left(i+\frac{1}{2}, j, k\right)=E_{2}^{n}\left(i, j+\frac{1}{2}, k\right)=0 & \text { for } k \in\{0, m\}
\end{array}
$$

and

$$
\begin{array}{ll}
H_{1}^{n}\left(i, j+\frac{1}{2}, k+\frac{1}{2}\right)=0 & \text { for } i \in\{0, m\}, \\
H_{2}^{n}\left(i+\frac{1}{2}, j, k+\frac{1}{2}\right)=0 & \text { for } j \in\{0, m\}, \\
H_{3}^{n}\left(i+\frac{1}{2}, j+\frac{1}{2}, k\right)=0 & \text { for } k \in\{0, m\} .
\end{array}
$$

This choice fits to the boundary conditions in (2), see Lemma 3.7. 
Example 1: Impact of the regularity of the initial data on the accuracy

In the first example we let $\varepsilon \equiv 1$ and $\mu \equiv 1$. It can be verified by straightforward calculations that each of the functions

$$
\begin{aligned}
u_{\kappa \lambda}^{1}(t, x)=\left(\begin{array}{c}
\sin \left(\kappa \pi x_{2}\right) \sin \left(\lambda \pi x_{3}\right) \cos \left(\sqrt{\kappa^{2}+\lambda^{2}} \pi t\right) \\
0 \\
0 \\
0 \\
u_{\kappa \lambda}^{2}(t, x)=\left(\begin{array}{c}
\frac{\lambda}{\sqrt{\kappa^{2}+\lambda^{2}}} \sin \left(\kappa \pi x_{2}\right) \cos \left(\lambda \pi x_{3}\right) \sin \left(\sqrt{\kappa^{2}+\lambda^{2}} \pi t\right) \\
\frac{\kappa}{\sqrt{\kappa^{2}+\lambda^{2}}} \cos \left(\kappa \pi x_{2}\right) \sin \left(\lambda \pi x_{3}\right) \sin \left(\sqrt{\kappa^{2}+\lambda^{2}} \pi t\right)
\end{array}\right), \\
0 \\
\frac{\lambda}{\sqrt{\kappa^{2}+\lambda^{2}}} \sin \left(\kappa \pi x_{1}\right) \sin \left(\lambda \pi x_{3}\right) \cos \left(\sqrt{\kappa^{2}+\lambda^{2}} \pi t\right) \\
0 \\
-\frac{\kappa}{\sqrt{\kappa^{2}+\lambda^{2}}} \cos \left(\lambda \pi x_{3}\right) \sin \left(\sqrt{\kappa^{2}+\lambda^{2}} \pi t\right) \\
0 \\
u_{\kappa \lambda}^{3}(t, x)=\left(\begin{array}{c}
0 \\
0 \\
-\frac{\lambda}{\sqrt{\kappa^{2}+\lambda^{2}}} \sin \left(\kappa \pi x_{1}\right) \sin \left(\lambda \pi x_{3}\right) \sin \left(\sqrt{\kappa^{2}+\lambda^{2}} \pi t\right)
\end{array}\right), \\
\frac{\kappa}{\sqrt{\kappa^{2}+\lambda^{2}}} \cos \left(\kappa \pi x_{1}\right) \sin \left(\lambda \pi x_{2}\right) \sin \left(\sqrt{\kappa^{2}+\lambda^{2}} \pi t\right) \\
0
\end{array}\right),
\end{aligned}
$$

with $(\kappa, \lambda) \in \mathbb{Z}^{2} \backslash\{(0,0)\}$ solves Maxwell's equations (2) including boundary and divergence conditions. More general solutions can be constructed by superposition

$$
u(t, x)=\sum_{\kappa=0}^{\kappa_{\max }} \sum_{\lambda=0}^{\lambda_{\max }}\left(a_{\kappa \lambda}^{1} u_{\kappa \lambda}^{1}(t, x)+a_{\kappa \lambda}^{2} u_{\kappa \lambda}^{2}(t, x)+a_{\kappa \lambda}^{3} u_{\kappa \lambda}^{3}(t, x)\right)
$$

with coefficients $a_{\kappa \lambda}^{\ell} \in \mathbb{R}$ and $a_{00}^{\ell}=0$ for $\ell \in\{1,2,3\}$. The initial conditions are obtained by simply evaluating (33) for $t=0$.

Numerical approximations were computed on the time-interval $[0,5]$ with different values of $\tau$ and $h$. For each combination, the spatial error at a fixed time is measured by the discrete counterpart of the norm $\|\cdot\|_{L^{2}}$, and for the global error we consider the maximum $L^{2}$-error over all time steps. In the first example, we let

$$
a_{11}^{1}=\gamma, \quad a_{11}^{2}=2 \gamma, \quad a_{11}^{3}=3 \gamma, \quad a_{\kappa \lambda}^{\ell}=0 \text { for all }(\kappa, \lambda) \neq(1,1)
$$

with a constant $\gamma$ chosen in such a way that $\|u(0, \cdot)\|_{L^{2}}=1$. The result is shown in the left picture of Figure 1 . For $\tau \geq 5 \cdot 2^{-9} \approx 0.0098$, the global error is dominated by the error of the time discretization. In perfect agreement with Theorem 4.5, we observe second-order convergence in time independently of 

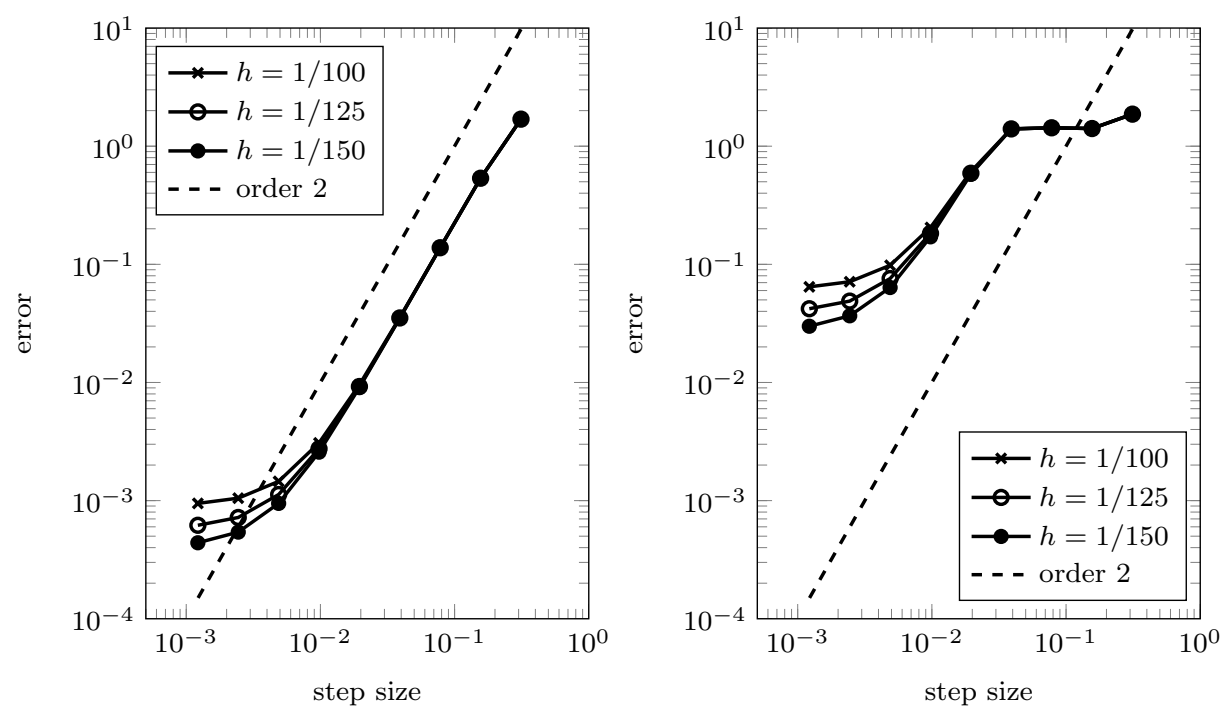

Fig. 1 Global error of the full discretization with step size $\tau=5 \cdot 2^{-k}$ in time $(k=4,5, \ldots, 12)$ and spatial mesh width $h=1 / 100,1 / 125,1 / 150$. The dashed line shows the function $\tau \rightarrow 100 \cdot \tau^{2}$ for the sake of comparison. For the coefficients in the exact solution (33) we have chosen (34) in the left panel and (35) in the right panel. In both cases, $\gamma$ was chosen in such a way that $\|u(0, \cdot)\|_{L^{2}}=1$.

the mesh width, i.e. independently of the norms of the discretization matrices. For $\tau<5 \cdot 2^{-9}$, the error of the space discretization starts to dominate the total error. As expected smaller values of $h$ yield higher accuracy.

According to Theorem 4.5, the error of the ADI method depends on the smoothness of the initial data. In order to illustrate this, the same numerical experiment is repeated with

$$
\begin{array}{lll}
a_{11}^{1}=\gamma, & a_{11}^{2}=2 \gamma, & a_{11}^{3}=3 \gamma, \\
a_{54}^{1}=3 \gamma, & a_{35}^{2}=2 \gamma, & a_{55}^{3}=\gamma
\end{array}
$$

and $a_{\kappa \lambda}^{\ell}=0$ for all other coefficients, where $\gamma$ is again chosen in such a way that $\|u(0, \cdot)\|_{L^{2}}=1$. In this example, the solution oscillates rapidly in space due to the terms corresponding to $a_{54}^{1}, a_{35}^{2}$, and $a_{55}^{3}$. The right picture in Figure 1 shows that the error does not explode, but that the convergence only starts for much smaller step sizes than before. The reason is that the term $\left\|M_{0}^{3} w\right\|_{L^{2}}$ with $w=u(0, \cdot)$ in Theorem 4.5 is now much larger due to the lower regularity of the initial data. The error plot also indicates that the step size where convergence starts $\left(\tau<5 \cdot 2^{-7} \approx 0.039\right)$ does not depend on the mesh width $h$. The reason is that the term $\left\|M_{0}^{3} w\right\|_{L^{2}}$ is independent of $h$. 


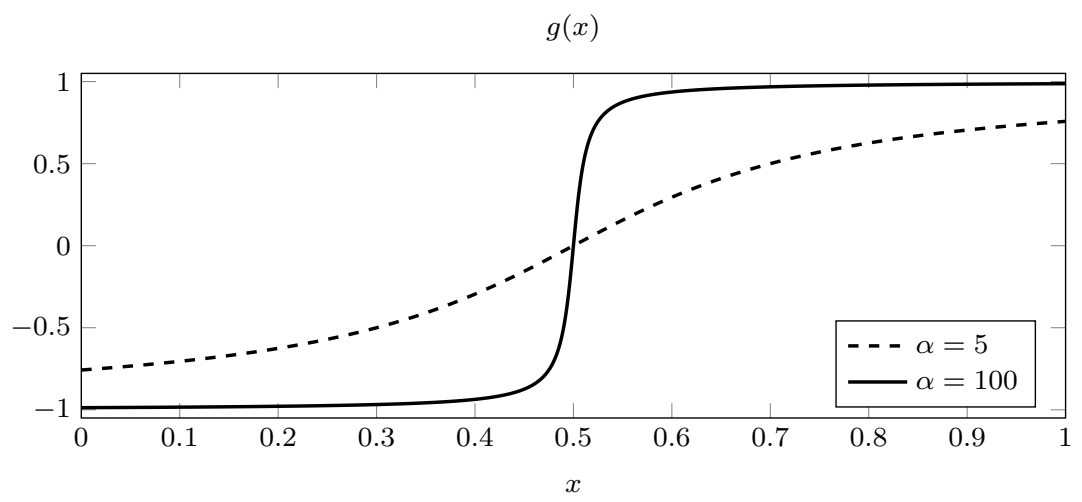

Fig. 2 Function $g\left(x_{i}\right)$ defined in (37).

Example 2: Impact of the regularity of the coefficients on the accuracy

In the second model problem we test how the accuracy is affected by the smoothness of the coefficient functions $\varepsilon$ and $\mu$. We let

$$
\begin{aligned}
\varepsilon(x) & =\mu(x)=2+g\left(x_{1}\right) g\left(x_{2}\right) g\left(x_{3}\right), \\
g\left(x_{i}\right) & =\frac{2}{\pi} \arctan \left(\alpha\left(x_{i}-0.5\right)\right), \quad i \in\{1,2,3\}
\end{aligned}
$$

and either $\alpha=5$ or $\alpha=100$. The function $g$ is depicted in Figure 2. For $\alpha=5$ both $\varepsilon(x)$ and $\mu(x)$ are so smooth that the convergence order two in time is not affected, which can be seen in Figure 3. For $\alpha=100$, the function $g\left(x_{i}\right)$ rapidly changes its value from -1 to 1 when $x_{i} \approx 0.5$, and Figure 3 shows that in this case the low regularity of $\varepsilon$ and $\mu$ spoils the order of convergence as expected. In this case, convergence of order two could only be observed for considerably smaller step sizes $\tau$ and a much smaller mesh width $h$. Since no explicit formula for the exact solution is available for our choice of $\varepsilon(x)$ and $\mu(x)$, the error of the time discretization was estimated by means of a reference solution which was computed with $\tau=5 \cdot 2^{-11}$ and $h=0.01$. For both values of $\alpha$, we have used the initial data

$$
\mathbf{E}(0, x)=\frac{\widetilde{\mathbf{E}}(0, x)}{\varepsilon(x)}, \quad \mathbf{H}(0, x)=\frac{\widetilde{\mathbf{H}}(0, x)}{\mu(x)}, \quad\left(\begin{array}{c}
\tilde{\mathbf{E}}(0, x) \\
\tilde{\mathbf{H}}(0, x)
\end{array}\right)=u(0, x)
$$

with $u$ defined in (33) and parameters

$$
a_{11}^{1}=a_{11}^{2}=a_{11}^{3}=1, \quad a_{\kappa \lambda}^{\ell}=0 \text { for all }(\kappa, \lambda) \neq(1,1) .
$$




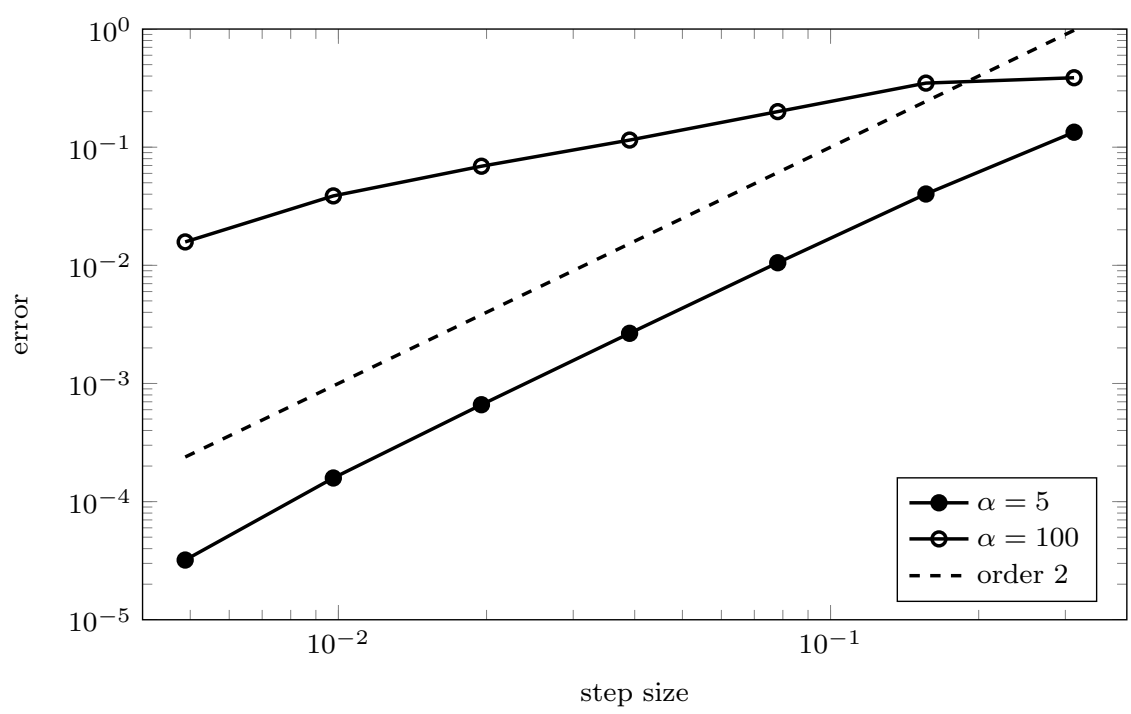

Fig. 3 Global error of the time discretization for non-constant coefficients (36), (37) with $\alpha=5$ (dots) and $\alpha=100$ (circles). Approximations were computed on the interval $[0,5]$ with step size $\tau=5 \cdot 2^{-k}(k=4,5, \ldots, 10)$ and spatial mesh width $h=1 / 100$. The dashed line shows the function $\tau \rightarrow 10 \cdot \tau^{2}$ for the sake of comparison.

\section{Appendix}

We now present two proofs of Lemmas 3.6 and 3.7 we omitted in Section 3.

Proof of Lemma 3.6. Lax-Milgram provides us with a unique $v \in H_{\Gamma}^{1}(Q)$ satisfying (21). To show the asserted regularity of $v$, we consider the operators $A_{j}=-\partial_{j}^{2}$ on $L^{2}(Q)$ whose domain consists of those $w \in L^{2}(Q)$ such that $\partial_{j}^{2} w \in L^{2}(Q), w=0$ on $\Gamma_{j}^{+}$or on $\Gamma_{j}^{-}$if $\Gamma_{j}^{+} \subseteq \Gamma$ or if $\Gamma_{j}^{-} \subseteq \Gamma$, respectively, and $\partial_{j} w=0$ on $\Gamma_{j}^{+}$or on $\Gamma_{j}^{-}$if $\Gamma_{j}^{+} \subseteq \Gamma^{\prime}$ or if $\Gamma_{j}^{-} \subseteq \Gamma^{\prime}$, respectively. Here and below we have $j=1,2,3$. For $u \in D\left(A_{j}\right)$ and $v \in D_{j}$, an integration by parts shows

$$
\int_{Q}\left(u v+A_{j} u v\right) \mathrm{d} x=\int_{Q}\left(u v+\partial_{j} u \partial_{j} v\right) \mathrm{d} x=: a(u, v),
$$

where $a$ is a symmetric, continuous and coercive bilinear form. It is routine to check that $A_{j}$ is the self adjoint operator induced by $a$. It is clear that $A_{j}$ is positive. In particular, $D_{j}$ is the domain of $A_{j}^{\frac{1}{2}}$ and hence $\partial_{j} A_{j}^{-\frac{1}{2}}$ is bounded on $L^{2}(Q)$.

To see that the resolvents of $A_{i}$ and $A_{j}$ commute, we observe that the resolvent of, say, $A_{1}$ is given by $\left(\left(\lambda I+A_{1}\right)^{-1} f\right)(x, y, z)=\left(R_{1}(\lambda) f(\cdot, y, z)\right)(x)$ for $\lambda>0$, for almost every $(x, y, z) \in Q$ and the resolvent $R_{1}(\lambda)$ of the 
negative second derivative on $L^{2}\left(a_{1}^{-}, a_{1}^{+}\right)$with the boundary conditions of $A_{1}$. Analogous facts hold for $A_{2}$ and $A_{3}$. If $f$ is the product of $f_{k} \in L^{2}\left(a_{k}^{-}, a_{k}^{+}\right)$for $k=1,2,3$, then $\left(\lambda I+A_{i}\right)^{-1}\left(\lambda I+A_{j}\right)^{-1} f=\left(\lambda I+A_{j}\right)^{-1}\left(\lambda I+A_{i}\right)^{-1} f$. Since the span of such functions is dense in $L^{2}(Q)$, the resolvents commute.

As explained in Sections III.4, VII.2 and X.1 of [21], we thus have a joint functional calculus with respect to $A_{1}, A_{2}$ and $A_{3}$ for Borel measurable functions $\phi: \mathbb{R}_{+}^{3} \rightarrow \mathbb{R}$. The operator $\phi\left(A_{1}, A_{2}, A_{3}\right)$ is bounded if $\phi$ is bounded, and for $h(\lambda)=1+\lambda_{1}+\lambda_{2}+\lambda_{3}$ we have $h\left(A_{1}, A_{2}, A_{3}\right)=I+A_{1}+A_{2}+A_{3}=: I+A$ on the domain $D(A):=D\left(A_{1}\right) \cap D\left(A_{2}\right) \cap D\left(A_{3}\right)$. Set $\rho=1 / h$. Then $\rho\left(A_{1}, A_{2}, A_{3}\right)$ is bounded and it is the inverse of $I+A$, so that $A$ is closed. Using the bounded functions $h_{i, j}(\lambda)=\lambda_{i}^{\frac{1}{2}} \lambda_{j}^{\frac{1}{2}} \rho(\lambda)$, we see that the operator $h_{i j}\left(A_{1}, A_{2}, A_{3}\right)=A_{i}^{\frac{1}{2}} A_{j}^{\frac{1}{2}}(I+A)^{-1}$ is bounded for all $i, j \in\{1,2,3\}$. This means that $D(A) \hookrightarrow H^{2}(Q)$ implying $D(A)=D$ and the equivalence of graph norm of $\Delta$ and the $H^{2}$-norm on $D$. It is then clear that $v=(I+A)^{-1} f$ is the required weak solution.

Proof of Lemma 3.7. 1) Throughout, let $(\mathbf{E}, \mathbf{H}) \in D\left(M_{0}^{2}\right)$. It is known that a map $u \in H($ rot $) \cap H\left(\right.$ div) belongs to $H^{1}(Q)^{3}$ if $u \times \nu=0$ or $u \cdot \nu=0$ holds on $\partial Q$. Moreover, the $H^{1}$ norm of $u$ is then dominated by $\|u\|_{L^{2}}+\|\operatorname{div} u\|_{L^{2}}+$ $\|\operatorname{rot} u\|_{L^{2}}$, see, e.g., Theorem 2.17 in [1]. Note that the equations (14) and (16) still hold on $Q$. In particular $\operatorname{div} \mathbf{E}$ and $\operatorname{div} \mathbf{H}$ belong to $L^{2}(Q)^{3}$. We thus have $\mathbf{E}, \mathbf{H} \in H^{1}(Q)^{3}$ and $\|(\mathbf{E}, \mathbf{H})\|_{H^{1}} \leq c\left(\|(\mathbf{E}, \mathbf{H})\|_{X}+\left\|M_{0}(\mathbf{E}, \mathbf{H})\right\|_{X}\right)$. The asserted zero-order traces for $\mathbf{E}$ and $\mathbf{H}$ now are a direct consequence of the boundary conditions $\mathbf{E} \times \nu=0$ and $\mathbf{H} \cdot \nu=0$, respectively.

Since $\mathbf{E}, \mathbf{H} \in H^{1}(Q)^{3} \hookrightarrow L^{6}(Q)^{3}$ and $M^{2}(\mathbf{E}, \mathbf{H}) \in X$, equation (16) and the assumptions on $\varepsilon$ and $\mu$ imply that $\Delta E_{j}, \Delta H_{j} \in L^{2}(Q)$. A standard localization argument then yields $E_{j}, H_{j} \in H_{\mathrm{loc}}^{2}(Q)^{3}$ for $j=1,2,3$. In addition, the $X$-norm of $(\Delta \mathbf{E}, \Delta \mathbf{H})$ is bounded by that of $M_{0}^{2}(\mathbf{E}, \mathbf{H})$ and $(\mathbf{E}, \mathbf{H})$. We next establish the properties of the traces of $\mathbf{E}$ and $\mathbf{H}$ needed to derive $\mathbf{E}, \mathbf{H} \in H^{2}(Q)^{3}$ from Lemma 3.6.

2) We first consider $E_{1}$. We will actually show that $\varepsilon E_{1}$ belongs to $H^{2}(Q)$ by applying Lemma 3.6 to $\varepsilon E_{1}$. Because of

$$
\partial_{k l} E_{1}=\frac{1}{\varepsilon} \partial_{k l}\left(\varepsilon E_{1}\right)-\frac{\partial_{k} \varepsilon}{\varepsilon} \partial_{l} E_{1}-\frac{\partial_{l} \varepsilon}{\varepsilon} \partial_{k} E_{1}-\frac{\partial_{k l} \varepsilon}{\varepsilon} E_{1},
$$

it will then follow that $E_{1} \in H^{2}(Q)$ employing $E_{1} \in H^{1}(Q)$ and the assumed regularity of $\varepsilon$. At the present stage, from (38), $\Delta E_{1} \in L^{2}(Q)$ and $E_{1} \in$ $H_{\text {loc }}^{2}(Q)^{3}$ we can already infer that $f:=(I-\Delta)\left(\varepsilon E_{1}\right) \in L^{2}(Q)$ and $\varepsilon E_{1} \in$ $H_{\text {loc }}^{2}(Q)$. Part 1) shows that $\varepsilon E_{1}=0$ on the faces $\Gamma:=\Gamma_{2}^{-} \cup \Gamma_{2}^{+} \cup \Gamma_{3}^{-} \cup \Gamma_{3}^{+}$. Fix a function $\psi \in H^{1}(Q)$ with $\partial_{2} \psi, \partial_{3} \psi \in H^{1}(Q)$ and having support in $\left[a_{1}^{-}, a_{1}^{+}\right] \times\left[a_{2}^{-}+\eta, a_{2}^{+}-\eta\right] \times\left[a_{3}^{-}+\eta, a_{3}^{+}-\eta\right]$ for some small $\eta=\eta(\psi)>0$. A given $\varphi \in H_{\Gamma}^{1}(Q)$ can be approximated in $H^{1}(Q)$ by such $\psi$ employing cutoff and mollification in the $\left(x_{2}, x_{3}\right)$ directions. For each sufficiently small $\kappa>0$, we set

$$
Q_{\kappa}=\left(a_{1}^{-}+\kappa, a_{1}^{+}-\kappa\right) \times\left(a_{2}^{-}+\kappa, a_{2}^{+}-\kappa\right) \times\left(a_{3}^{-}+\kappa, a_{3}^{+}-\kappa\right) .
$$


We take $\kappa \in(0, \eta(\psi))$ and denote by $\Gamma_{1}^{ \pm}(\kappa)$ the open faces of $Q_{\kappa}$ containing points of the form $\left(a_{1}^{ \pm} \pm \kappa, x_{2}, x_{3}\right)$. Integrating by parts and using $\operatorname{div}(\varepsilon \mathbf{E})=0$ as well as $\partial_{j}\left(\varepsilon E_{j}\right) \in H_{\text {loc }}^{1}(Q)$ for $j=1,2,3$, we conclude that

$$
\begin{aligned}
\int_{Q} \nabla\left(\varepsilon E_{1}\right) \cdot \nabla \psi \mathrm{d} x & +\int_{Q} \varepsilon E_{1} \psi \mathrm{d} x=\lim _{\kappa \rightarrow 0} \int_{Q_{\kappa}}\left(\varepsilon E_{1} \psi+\nabla\left(\varepsilon E_{1}\right) \cdot \nabla \psi\right) \mathrm{d} x \\
& =\lim _{\kappa \rightarrow 0}\left[\int_{Q_{\kappa}}(I-\Delta)\left(\varepsilon E_{1}\right) \psi \mathrm{d} x+\int_{\partial Q_{\kappa}} \psi \nabla\left(\varepsilon E_{1}\right) \cdot \nu \mathrm{d} \sigma\right] \\
& =\int_{Q} f \psi \mathrm{d} x \pm \lim _{\kappa \rightarrow 0} \int_{\Gamma_{1}^{ \pm}(\kappa)} \psi \partial_{1}\left(\varepsilon E_{1}\right) \mathrm{d}\left(x_{2}, x_{3}\right) \\
& =\int_{Q} f \psi \mathrm{d} x \mp \lim _{\kappa \rightarrow 0} \int_{\Gamma_{1}^{ \pm}(\kappa)} \psi\left(\partial_{2}\left(\varepsilon E_{2}\right)+\partial_{3}\left(\varepsilon E_{3}\right)\right) \mathrm{d}\left(x_{2}, x_{3}\right) \\
& =\int_{Q} f \psi \mathrm{d} x \pm \lim _{\kappa \rightarrow 0} \int_{\Gamma_{1}^{ \pm}(\kappa)}\left(\varepsilon E_{2} \partial_{2} \psi+\varepsilon E_{3} \partial_{3} \psi\right) \mathrm{d}\left(x_{2}, x_{3}\right) \\
& =\int_{Q} f \psi \mathrm{d} x .
\end{aligned}
$$

We have used that $\psi$ vanishes near $\Gamma$ for the penultimate equation and that $\varepsilon E_{j}, \partial_{j} \psi \in H^{1}(Q)^{3}$ and $\varepsilon E_{j}=0$ on $\Gamma_{1}^{ \pm}$for $j=2,3$ in the last identity, see part 1). By approximation, equation (39) then holds for all $\psi \in H_{\Gamma}^{1}(Q)$, and hence Lemma 3.6 yields $\varepsilon E_{1} \in H^{2}(Q)$ so that $E_{1} \in H^{2}(Q)$ as explained above. In the same way, one sees that $E_{2}, E_{3} \in H^{2}(Q)$. Moreover, $\left\|E_{j}\right\|_{H^{2}}$ is bounded by $c\left(\left\|E_{j}\right\|_{L^{2}}+\left\|\Delta E_{j}\right\|_{L^{2}}\right)$ due to Lemma 3.6 and hence by $c\left(\|(\mathbf{E}, \mathbf{H})\|_{X}+\right.$ $\left.\left\|M_{0}^{2}(\mathbf{E}, \mathbf{H})\right\|_{X}\right)$ in view of step 1).

We denote by $\gamma_{i}$ the trace operator to $\Gamma_{i}^{ \pm}$, where $i, j, k \in\{1,2,3\}$. Since $E_{k} \in H^{2}(Q)$, one can approximate $E_{k}$ in $H^{2}(Q)$ by $v_{n} \in C^{2}(\bar{Q})$. Clearly, $\gamma_{i} \partial_{j} v_{n}=\partial_{j} \gamma_{i} v_{n}$ and thus $\gamma_{i} \partial_{j} E_{k}=\partial_{j} \gamma_{i} E_{k}$. As a result, the asserted first order boundary conditions of $\mathbf{E}$ follow from the already established 0-order boundary conditions of $\mathbf{E}$.

3) Next, we consider $H_{1}$ and set $g:=(I-\Delta) H_{1} \in L^{2}(Q)$. Here we have less Dirichlet boundary conditions, namely $H_{j}=0$ on $\Gamma_{j}^{ \pm}$for $j=1,2,3$. To deal with the Neumann conditions, we first note that

$$
\begin{aligned}
& \operatorname{rot}\left(\varepsilon^{-1} \operatorname{rot} \mathbf{H}\right) \in L^{2}(Q)^{3}, \quad \varepsilon^{-1} \operatorname{rot} \mathbf{H} \times \nu=0 \text { on } \partial Q, \\
& \operatorname{div}\left(\varepsilon^{-1} \operatorname{rot} \mathbf{H}\right)=\nabla \varepsilon^{-1} \cdot \operatorname{rot} \mathbf{H} \in L^{2}(Q) .
\end{aligned}
$$

Hence, $\varepsilon^{-1} \operatorname{rot} \mathbf{H}$ belongs to $H^{1}(Q)^{3}$ which yields $\operatorname{rot} \mathbf{H} \in H^{1}(Q)^{3}$. It also follows that $\operatorname{rot} \mathbf{H} \times \nu=0$ on $\partial Q$. In particular, the first component of $\operatorname{rot} \mathbf{H}$ vanishes on $\Gamma_{2}^{ \pm} \cup \Gamma_{3}^{ \pm}$.

We set $\tilde{\Gamma} \stackrel{2}{=} \Gamma_{1}^{-} \cup \Gamma_{1}^{+}$and define the faces $\Gamma_{j}^{ \pm}(\kappa)$ of $Q_{\kappa}$ in the $j$ th direction for $j=2,3$, cf. step 2). We take functions $\psi \in H^{1}(Q)$ with $\partial_{1} \psi \in H^{1}(Q)$ and having support in $\left[a_{1}^{-}+\eta, a_{1}^{+}-\eta\right] \times\left[a_{2}^{-}, a_{2}^{+}\right] \times\left[a_{3}^{-}, a_{3}^{+}\right]$for some $\eta>0$. We choose $\kappa \in(0, \eta)$ so that $\psi$ vanishes around $\Gamma_{1}^{ \pm}(\kappa)$. As above, we deduce

$$
\int_{Q} \nabla H_{1} \cdot \nabla \psi \mathrm{d} x+\int_{Q} H_{1} \psi \mathrm{d} x=\lim _{\kappa \rightarrow 0} \int_{Q_{\kappa}}\left(H_{1} \psi+\nabla H_{1} \cdot \nabla \psi\right) \mathrm{d} x
$$




$$
\begin{aligned}
= & \lim _{\kappa \rightarrow 0}\left[\int_{Q_{\kappa}} \psi(I-\Delta) H_{1} \mathrm{~d} x+\int_{\partial Q_{\kappa}} \psi \nu \cdot \nabla H_{1} \mathrm{~d} \sigma\right] \\
= & \int_{Q} \psi(I-\Delta) H_{1} \mathrm{~d} x+\lim _{\kappa \rightarrow 0} \int_{\partial Q_{\kappa}}\left[\psi \nu \cdot \nabla H_{1}-(\operatorname{rot} \mathbf{H} \times \nu) \cdot(\psi, 0,0)\right] \mathrm{d} \sigma \\
= & \int_{Q} g \psi \mathrm{d} x+\lim _{\kappa \rightarrow 0} \int_{\partial Q_{\kappa}} \psi \nu \partial_{1} \mathbf{H} \mathrm{d} \sigma \\
= & \int_{Q} g \psi \mathrm{d} x \pm \lim _{\kappa \rightarrow 0}\left[\int_{\Gamma_{2}^{ \pm}(\kappa)} \psi \partial_{1} H_{2} \mathrm{~d} \sigma+\int_{\Gamma_{3}^{ \pm}(\kappa)} \psi \partial_{1} H_{3} \mathrm{~d} \sigma\right] \\
= & \int_{Q} g \psi \mathrm{d} x \mp \lim _{\kappa \rightarrow 0}\left[\int_{\Gamma_{2}^{ \pm}(\kappa)} H_{2} \partial_{1} \psi \mathrm{d} \sigma+\int_{\Gamma_{3}^{ \pm}(\kappa)} H_{3} \partial_{1} \psi \mathrm{d} \sigma\right] \\
= & \int_{Q} g \psi \mathrm{d} x .
\end{aligned}
$$

The remaining assertions now follow as in step 2).

\section{References}

1. C. Amrouche, C. Bernardi, M. Dauge, and V. Girault. Vector potentials in threedimensional non-smooth domains. Mathematical Methods in the Applied Sciences, 21 (9):823-864, 1998.

2. W. Chen, X. Li, and D. Liang. Energy-conserved splitting FDTD methods for Maxwell's equations. Numer. Math., 108(3):445-485, 2008.

3. M. Costabel and M. Dauge. Singularities of electromagnetic fields in polyhedral domains. Archive for Rational Mechanics and Analysis, 151(3):221-276, 2000.

4. R. Dautray and J.-L. Lions. Mathematical analysis and numerical methods for science and technology. Volume 3: Spectral theory and applications. Springer, Berlin, 2nd edition, 2000. With the collaboration of Michel Artola and Michel Cessenat.

5. R. Dautray and J.-L. Lions. Mathematical analysis and numerical methods for science and technology. Volume 5: Evolution problems. I. Springer, Berlin, 2nd edition, 2000. With the collaboration of Michel Artola, Michel Cessenat and Hélène Lanchon.

6. K.-J. Engel and R. Nagel. One-parameter semigroups for linear evolution equations., volume 194 of Graduate Texts in Mathematics. Springer, Berlin, 2000.

7. I. Faragó, R. Horváth, and W.H.A. Schilders. Investigation of numerical timeintegrations of Maxwell's equations using the staggered grid spatial discretization. International Journal of Numerical Modelling : Electronic Networks, Devices and Fields, 18(2):149-169, 2005.

8. L. Gao, B. Zhang, and D. Liang. The splitting finite-difference time-domain methods for Maxwell's equations in two dimensions. J. Comput. Appl. Math., 205(1):207-230, 2007.

9. S. G. Garcia, Tae-Woo Lee, and S.C. Hagness. On the accuracy of the ADI-FDTD method. IEEE Antennas and Wireless Propagation Letters, 1(1):31 -34, 2002.

10. S. Gonzáles García, A. Rubio Bretones, R. Gómez Martín, and Susan C. Hagness. Accurate implementation of current sources in the ADI-FDTD scheme. IEEE Antennas and Wireless Propagation Letters, 2004.

11. S. Gonzáles García, R. Godoy Rubio, A. Rubio Bretones, and R. Gómez Martín. On the dispersion relation of ADI-FDTD. IEEE Microwave and Wireless Components Letters, 16(6):354-356, 2006.

12. E. Hairer, Ch. Lubich, and G. Wanner. Geometric Numerical Integration, StructurePreserving Algorithms for Ordinary Differential Equations, volume 31 of Springer Series in Computational Mathematics. Springer, Berlin, Heidelberg, 2006. 
13. E. Hansen and A. Ostermann. Dimension splitting for evolution equations. Numerische Mathematik, 108:557-570, 2008.

14. J. Lee and B. Fornberg. A split step approach for the 3-D Maxwell's equations. $J$. Comput. Appl. Math., 158(2):485-505, 2003.

15. J. Lee and B. Fornberg. Some unconditionally stable time stepping methods for the 3D Maxwell's equations. J. Comput. Appl. Math., 166(2):497-523, 2004.

16. R. Leis. Initial-boundary value problems in mathematical physics. B. G. Teubner, Stuttgart, 1986.

17. T. Namiki. A new FDTD algorithm based on alternating-direction implicit method IEEE Transactions on Microwave Theory and Techniques, 47(10):2003-2007, oct 1999.

18. T. Namiki. 3-D ADI-FDTD method-unconditionally stable time-domain algorithm for solving full vector Maxwell's equations. IEEE Transactions on Microwave Theory and Techniques, 48(10):1743-1748, oct 2000.

19. J. Rauch. Partial differential equations, volume 128 of Graduate Texts in Mathematics. Springer-Verlag, New York, 1991.

20. M. Reed and B. Simon. Methods of modern mathematical physics. I. Academic Press, Inc. [Harcourt Brace Jovanovich, Publishers], New York, second edition, 1980. Functional analysis.

21. B. Sz.-Nagy. Spektraldarstellung linearer Transformationen des Hilbertschen Raumes. Berichtigter Nachdruck, volume 39 of Ergebnisse der Mathematik und ihrer Grenzgebiete. Springer-Verlag, Berlin, New York, reprint of the 1942 original edition edition, 1967

22. A. Taflove and S. C. Hagness. Computational Electrodynamics: The Finite-Difference Time-Domain Method. Artech House Publishers, Norwood, 3rd edition, 2005.

23. M. Tucsnak and G. Weiss. Observation and control for operator semigroups. Birkhäuser, Basel, 2009.

24. J. G. Verwer. Component splitting for semi-discrete Maxwell equations. BIT, 51(2): 427-445, 2011.

25. J. G. Verwer. Composition methods, Maxwell's equations, and source terms. SIAM J. Numer. Anal., 50(2):439-457, 2012.

26. J. G. Verwer and M. A. Botchev. Unconditionally stable integration of Maxwell's equations. Linear Algebra Appl., 431(3-4):300-317, 2009.

27. K. Yee. Numerical solution of initial boundary value problems involving Maxwell's equations in isotropic media. IEEE Transactions on Antennas and Propagation, 14(3) $302-307,1966$.

28. F. Zheng, Z. Chen, and J. Zhang. Toward the development of a three-dimensional unconditionally stable finite-difference time-domain method. IEEE Transactions on Microwave Theory and Techniques, 48(9):1550-1558, sep 2000. 